\title{
Multilocus genetics to reconstruct aeromonad evolution
}

Frédéric Roger ${ }^{1}$, Hélène Marchandin ${ }^{1,2}$, Estelle Jumas-Bilak ${ }^{1,3}$, Angeli Kodjo ${ }^{4,5}$, the colBVH study group ${ }^{6}$ and Brigitte Lamy ${ }^{1,2,5,6^{*}}$

\begin{abstract}
Background: Aeromonas spp. are versatile bacteria that exhibit a wide variety of lifestyles. In an attempt to improve the understanding of human aeromonosis, we investigated whether clinical isolates displayed specific characteristics in terms of genetic diversity, population structure and mode of evolution among Aeromonas spp. A collection of 195 Aeromonas isolates from human, animal and environmental sources was therefore genotyped using multilocus sequence analysis (MLSA) based on the dnaK, gltA, gyrB, radA, rpoB, tsf and zipA genes.

Results: The MLSA showed a high level of genetic diversity among the population, and multilocus-based phylogenetic analysis (MLPA) revealed 3 major clades: the A. veronii, A. hydrophila and A. caviae clades, among the eleven clades detected. Lower genetic diversity was observed within the A. caviae clade as well as among clinical isolates compared to environmental isolates. Clonal complexes, each of which included a limited number of strains, mainly corresponded to host-associated subsclusters of strains, i.e., a fish-associated subset within A. salmonicida and 11 human-associated subsets, 9 of which included only disease-associated strains. The population structure was shown to be clonal, with modes of evolution that involved mutations in general and recombination events locally. Recombination was detected in 5 genes in the MLSA scheme and concerned approximately $50 \%$ of the STs.

Therefore, these recombination events could explain the observed phylogenetic incongruities and low robustness. However, the MLPA globally confirmed the current systematics of the genus Aeromonas.

Conclusions: Evolution in the genus Aeromonas has resulted in exceptionally high genetic diversity. Emerging from this diversity, subsets of strains appeared to be host adapted and/or "disease specialized" while the A. caviae clade displayed an atypical tempo of evolution among aeromonads. Considering that $A$. salmonicida has been described as a genetically uniform pathogen that has adapted to fish through evolution from a variable ancestral population, we hypothesize that the population structure of aeromonads described herein suggested an ongoing process of adaptation to specialized niches associated with different degrees of advancement according to clades and clusters.
\end{abstract}

\section{Background}

Aeromonads are ubiquitous free-living organisms found in aquatic environments with a strong ability to quickly colonize an exceptionally wide variety of habitats and hosts, ranging from hostile environments, such as polluted or chlorinated water, to leeches, insects, fish, mollusks,

\footnotetext{
* Correspondence: brigitte_lamy@yahoo.fr

${ }^{1}$ Laboratoire de Bactériologie-Virologie (UMR 5119 - Equipe Pathogènes et Environnements), Université Montpellier 1, 15, Avenue Charles Flahault, BP 14491, 34093 Montpellier Cedex 5, France

${ }^{2}$ Laboratoire de Bactériologie, Centre Hospitalier Régional Universitaire de Montpellier (Hôpital Arnaud de Villeneuve), 371, Avenue du Doyen Gaston Giraud, 34295 Montpellier Cedex 5, France

Full list of author information is available at the end of the article
}

and mammals, including man [1]. They are opportunistic pathogens involved in various types of infections in a wide range of hosts. This versatility is supported by a large variety of genes involved in metabolic fitness and virulence; thus, Aeromonas hydrophila is referred to as a "jack-of-alltrades" [2]. Despite the adaptability of A. hydrophila, very few mobile genetic elements, which are usually associated with rapid adaptation, have been found in the complete genomic sequence of the pathogenic strain A. hydrophila ATCC $7966^{\mathrm{T}}$ [2].

Additionally, because some hosts may only be either colonized or infected, the concept that only specific subsets of Aeromonas strains within species might actually be pathogenic for humans was proposed [3,4]. In this setting, 
the question has arisen of whether isolates causing infectious diseases are exceptional and can be distinguished from other strains. Comparative analyses including environmental and clinical isolates showed that clinical strains are well differentiated from strains collected in the environment based on multilocus enzyme electrophoresis (MLEE) [5]. Other studies employing phenotypic, genotypic and virulence analyses have failed to distinguish isolates involved in infectious diseases from those that are not $[3,6-8]$. However, this situation is complex because the pathogenesis of Aeromonas infection is multifactorial and is associated with multiple sources of variability (e.g., a wide variety of virulence factor genes and the influence of environmental conditions); these studies are usually limited either by the sampling strategy applied (e.g., including a low number of isolates, species or types of infection), incomplete virulence factor analyses, or an absence of virulence gene expression analysis.

Overall, in the case of generalist opportunistic pathogens, which do not meet all of the criteria Koch's postulate, the link between virulence-related genes and infection is not clearly established, and this opportunistic pathogenic behavior may instead be considered to represent an adaptation to human ecology [9-11]. There is evidence that genetic clusters can correspond to ecologically distinct populations and/or host-adapted populations, even when genes that are not related to virulence are considered [9,11-14].

In this context, in an attempt to improve the understanding of human aeromonosis, we investigated whether clinical isolates displayed specific characteristics among a large population of Aeromonas spp. from various origins. Because the 3 main Aeromonas species recovered from human clinical infectious diseases are A. caviae, A. hydrophila and $A$. veronii biovar sobria, we particularly focused on isolates belonging to these 3 taxa. The aim of this work was to determine the genetic characteristics, population structure and mode of evolution in a large population of aeromonads using a comparative approach that examined human, nonhuman animal and environmental strains. For this purpose, we developed a multilocus sequence analysis (MLSA) scheme specific for aeromonads, representing the third MLSA scheme to be described for this genus $[15,16]$. This strategy provided 4 new genes and produced new information on the mode of evolution, recombination rates and horizontal gene transfer in these species. This study, which was based on a large human clinical strain collection, provides interesting insight regarding the mode of evolution of aeromonads linked with human infection.

\section{Methods}

\section{Bacterial strains}

A total of 195 strains of Aeromonas spp., including 62 type and reference strains, were analyzed. The distribution of the origin of these strains was as follows: 115 human clinical strains, 39 non-human animal strains and 41 environmental strains (Table 1). Of the 115 human clinical isolates, 67 and 7 isolates were sampled in 2006 during a prospective study on aeromonosis involving 70 hospitals from mainland France and overseas territories, respectively [17]; 3 and 6 additional strains were isolated prior to 2006 from mainland France and overseas territories, respectively; 19 isolates were recovered from patients admitted to the Montpellier University Hospital between 2008 and 2010; and 5 strains were isolated in the USA, 2 in India, 2 in Taiwan, 1 in Bangladesh, 1 in New Zealand, 1 in Switzerland and 1 in the Czech republic. Clinical data were collected with the aim of specifying the clinical implications of the strains, i.e., whether they were involved in an infectious process or in host colonization. Of the 80 nonhuman isolates, 33 were isolated in France from a wastewater treatment lagoon $(\mathrm{n}=11)$, recreational lake water $(\mathrm{n}=13)$, healthy snails $(\mathrm{n}=7)$ or fish $(\mathrm{n}=2)$, and 36 strains were isolated from diverse sources among 13 countries worldwide, mainly in Europe and North America, while the countries of origin of 11 strains were unknown. The strain collection included type strains of 28 species and a representative strain of hybridization group (HG) 11 (Table 1).

\section{Pulsed-field gel electrophoresis (PFGE)-restriction fragment length polymorphism (RFLP) analysis}

Genomic DNA was prepared in agarose plugs as previously described [18] starting from a fresh culture on Trypticase Soja agar medium. After Aeromonas suspensions in $2 \mathrm{ml}$ of Tris- $\mathrm{NaCl}$ buffer (1.0 M Tris base, $1.0 \mathrm{M} \mathrm{NaCl}, \mathrm{pH}$ 7.6) were adjusted to an optical density of 1.5 at $650 \mathrm{~nm}$, they were centrifuged $(10,000 \mathrm{~g}$ for $1 \mathrm{~min}), 1 \mathrm{ml}$ of the supernatant was then discarded, and the pellet was resuspended (final concentration 2:1). DNA was digested at $25^{\circ} \mathrm{C}$ with $40 \mathrm{U}$ of SwaI (New England BioLabs, Hertfordshire, United Kingdom). The SwaI fragments were separated in a $1 \%$ agarose gel via PFGE using a CHEF-DRIII apparatus (BioRad Laboratories, Hercules, $\mathrm{CA}$ ) and 0.5X Tris-BorateEDTA (TBE) buffer containing $50 \mu \mathrm{M}$ thiourea at $5.5 \mathrm{~V} / \mathrm{cm}$ and $10^{\circ} \mathrm{C}$ with pulse ramps of 100 to $5 \mathrm{~s}$ for $48 \mathrm{~h}$. A lambda concatemer (Biolabs) was used as the size standard. The gel was stained with ethidium bromide and photographed under UV light. The PFGE profiles, known as pulsotypes, were compared visually by numbering both the shared and the distinct DNA fragments.

\section{Gene amplification and sequencing}

The complete genomic sequences of $A$. hydrophila subsp. hydrophila ATCC $7966^{\mathrm{T}}$ and A. salmonicida subsp. salmonicida A449 [GenBank accession numbers NC_008570 and NC_009348, respectively] were used employed as references for gene selection and primer design. The 
Table 1 Typing data and origin of Aeromonas strains analyzed in the study

\begin{tabular}{|c|c|c|c|c|c|c|c|c|c|c|c|c|c|c|}
\hline \multirow{2}{*}{$\begin{array}{l}\text { MLPA clade or } \\
\text { isolated taxon }\end{array}$} & \multirow[t]{2}{*}{ Strain } & \multirow[t]{2}{*}{ ST } & \multirow{2}{*}{$\begin{array}{l}\mathrm{CC} \\
(5)^{\mathrm{a}}\end{array}$} & \multirow{2}{*}{$\begin{array}{l}\mathrm{CC} \\
(4)^{\mathrm{b}}\end{array}$} & \multicolumn{7}{|c|}{ Allelic profile } & \multirow[t]{2}{*}{ Origin } & \multirow{2}{*}{$\begin{array}{l}\text { Infection (I) or } \\
\text { colonization (C) }\end{array}$} & \multirow{2}{*}{$\begin{array}{l}\text { Region, country } \\
\text { and year of isolation }\end{array}$} \\
\hline & & & & & dnaK & gltA & gyrB & $\operatorname{radA}$ & $r p o B$ & tsf & zipA & & & \\
\hline \multirow[t]{25}{*}{ A. hydrophila $(\mathrm{n}=35)$} & BVH3 & 2 & - & $A$ & 2 & 2 & 2 & 2 & 2 & 2 & 2 & Human, Wound & । & Cahors, Fr, 2006 \\
\hline & BVH14 & 7 & - & A & 7 & 2 & 7 & 7 & 2 & 2 & 2 & Human, Wound & । & Le Mans, Fr, 2006 \\
\hline & $\begin{array}{l}\text { A. hydrophila subsp. } \\
\text { hydrophila CCM } 2278\end{array}$ & 7 & - & A & 7 & 2 & 7 & 7 & 2 & 2 & 2 & $\begin{array}{l}\text { Non-human, } \\
\text { Red-legged Frog }\end{array}$ & । & California, USA, 1963 \\
\hline & BVH29 & 18 & - & - & 18 & 15 & 17 & 17 & 13 & 15 & 17 & Human, Wound & I & $\begin{array}{l}\text { Reunion Island, } \\
\text { Fr, } 2006\end{array}$ \\
\hline & BVH30 & 18 & - & - & 18 & 15 & 17 & 17 & 13 & 15 & 17 & Human, Stool & I & Langres, Fr, 2006 \\
\hline & BVH35 & 23 & - & - & 23 & 19 & 21 & 22 & 18 & 12 & 20 & Human, Wound & $C$ & $\begin{array}{l}\text { La Roche sur Yon, } \\
\text { Fr, } 2006\end{array}$ \\
\hline & BVH99 & 23 & - & - & 23 & 19 & 21 & 22 & 18 & 12 & 20 & Human, NA & ND & Brest, Fr, ND \\
\hline & BVH25a & 15 & - & - & 15 & 13 & 14 & 14 & 10 & 13 & 14 & $\begin{array}{l}\text { Human, Respiratory } \\
\text { tract }\end{array}$ & । & Saint-Brieux, Fr,2006 \\
\hline & BVH27b & 15 & - & - & 15 & 13 & 14 & 14 & 10 & 13 & 14 & Human, Wound & I & $\begin{array}{l}\text { Reunion Island, } \\
\text { Fr, } 2006\end{array}$ \\
\hline & AK204 & 15 & - & - & 15 & 13 & 14 & 14 & 10 & 13 & 14 & Non-human, Snail & । & Angers, Fr, 1995 \\
\hline & $\mathrm{BVH} 2$ & 1 & - & - & 1 & 1 & 1 & 1 & 1 & 1 & 1 & Human, Wound & 1 & Cahors, Fr, 2006 \\
\hline & BVH12 & 5 & - & - & 5 & 5 & 5 & 5 & 5 & 5 & 5 & $\begin{array}{l}\text { Human, Eyes } \\
\text { infection }\end{array}$ & 1 & Cherbourg, Fr, 2006 \\
\hline & $\mathrm{BVH} 24$ & 14 & - & - & 14 & 12 & 13 & 13 & 9 & 12 & 13 & Human, Wound & I & Saint-Brieux, Fr,2006 \\
\hline & BVH33 & 21 & - & - & 21 & 18 & 20 & 20 & 16 & 2 & 20 & Human, Wound & । & La Rochelle, Fr, 2006 \\
\hline & BVH34 & 22 & - & - & 22 & 15 & 17 & 21 & 17 & 18 & 21 & Human, Urine & $C$ & $\begin{array}{l}\text { La Roche sur Yon, } \\
\text { Fr, } 2006\end{array}$ \\
\hline & BVH36 & 24 & - & - & 24 & 20 & 22 & 23 & 19 & 5 & 22 & Human, Wound & । & $\begin{array}{l}\text { La Roche sur Yon, } \\
\text { Fr, } 2006\end{array}$ \\
\hline & $\mathrm{BVH} 41$ & 28 & - & - & 28 & 24 & 26 & 27 & 23 & 22 & 26 & Human, Wound & । & Vannes, Fr, 2006 \\
\hline & $\mathrm{BVH} 42$ & 29 & - & - & 29 & 25 & 27 & 28 & 24 & 12 & 27 & Human, Wound & I & Périgueux, Fr, 2006 \\
\hline & $\mathrm{BVH} 45$ & 32 & - & - & 32 & 28 & 30 & 14 & 10 & 25 & 30 & Human, Wound & । & Périgueux, Fr, 2006 \\
\hline & BVH64 & 48 & - & - & 47 & 15 & 44 & 42 & 35 & 35 & 42 & Human, Wound & । & Belfort, Fr, 2006 \\
\hline & BVH72 & 55 & - & - & 52 & 42 & 49 & 48 & 39 & 12 & 46 & Human, Blood & ND & $\begin{array}{l}\text { Martinique Island, } \\
\mathrm{Fr}, \mathrm{ND}\end{array}$ \\
\hline & BVH75 & 57 & - & - & 53 & 44 & 51 & 49 & 2 & 12 & 48 & $\begin{array}{l}\text { Human, Respiratory } \\
\text { tract }\end{array}$ & । & Saint-Etienne, Fr,2006 \\
\hline & BVH93 & 68 & - & - & 63 & 52 & 60 & 57 & 47 & 45 & 14 & Human, Wound & । & Cahors, Fr, 2006 \\
\hline & BVH96 & 70 & - & - & 65 & 54 & 62 & 59 & 48 & 47 & 56 & Human, Wound & C & Bourg en Bresse, Fr, 2006 \\
\hline & BVH97 & 71 & - & - & 66 & 55 & 63 & 1 & 49 & 48 & 57 & Human, Wound & । & $\begin{array}{l}\text { Bourg en Bresse, } \\
\text { Fr, } 2006\end{array}$ \\
\hline
\end{tabular}


Table 1 Typing data and origin of Aeromonas strains analyzed in the study (Continued)

\begin{tabular}{|c|c|c|c|c|c|c|c|c|c|c|c|c|c|c|}
\hline & ADV105 & 76 & - & - & 69 & 58 & 67 & 63 & 51 & 49 & 60 & Human, Stool & ND & Montpellier, Fr, 2008 \\
\hline & AK203 & 93 & - & - & 86 & 70 & 81 & 76 & 60 & 61 & 14 & Non-human, Snail & । & Angers, Fr, 1995 \\
\hline & AK218 & 95 & - & - & 88 & 72 & 83 & 78 & 47 & 49 & 74 & $\begin{array}{l}\text { Environment, Waste } \\
\text { water treatment } \\
\text { lagoon }\end{array}$ & - & Montracol, Fr, 2006 \\
\hline & AK235 & 105 & - & - & 97 & 79 & 93 & 88 & 66 & 67 & 81 & $\begin{array}{l}\text { Environment, Waste } \\
\text { water treatment } \\
\text { lagoon }\end{array}$ & - & Montracol, Fr, 2006 \\
\hline & $\begin{array}{l}\text { A. hydrophila subsp. } \\
\text { hydrophila CECT } 839^{\top}\end{array}$ & 130 & - & - & 120 & 102 & 114 & 108 & 79 & 81 & 22 & $\begin{array}{l}\text { Environment, Tin of } \\
\text { milk with a fishy } \\
\text { odor }\end{array}$ & - & $N A, N A, N A$ \\
\hline & $\begin{array}{l}\text { A. hydrophila subsp. } \\
\text { ranae CIP } 107985\end{array}$ & 131 & - & - & 121 & 103 & 115 & 109 & 80 & 82 & 101 & Non-human, Frog & । & NA, Thaïland, NA \\
\hline & $\begin{array}{l}\text { A. hydrophila } \\
\text { CECT } 5734\end{array}$ & 163 & - & - & 150 & 132 & 144 & 137 & 104 & 12 & 127 & Non-human, Fish & I & Valencia, Spain, 1987 \\
\hline & $\begin{array}{l}\text { A. hydrophila subsp. } \\
\text { hydrophila CCM } 2280\end{array}$ & 171 & - & - & 69 & 139 & 152 & 145 & 111 & 115 & 134 & Non-human, Snake & - & NA, NA, 1963 \\
\hline & $\begin{array}{l}\text { A. hydrophila subsp. } \\
\text { hydrophila CCM } 2282\end{array}$ & 172 & - & - & 158 & 140 & 153 & 146 & 47 & 116 & 135 & $\begin{array}{l}\text { Non-human, } \\
\text { Nile Monitor }\end{array}$ & ND & NA, NA, 1963 \\
\hline & $\begin{array}{l}\text { A. hydrophila subsp. } \\
\text { hydrophila CCM } 4528\end{array}$ & 174 & - & - & 160 & 15 & 17 & 148 & 13 & 118 & 137 & Human, Stool & ND & $\begin{array}{l}\text { NA, Czech Republic, } \\
1993\end{array}$ \\
\hline \multirow[t]{13}{*}{ A. veronii $(n=71)$} & $\mathrm{BVH} 22$ & 13 & - & - & 13 & 11 & 12 & 4 & 8 & 11 & 12 & Human, Wound & I & Alès, Fr, 2006 \\
\hline & $\mathrm{BVH} 23$ & 13 & - & - & 13 & 11 & 12 & 4 & 8 & 11 & 12 & Human, Wound & । & Saint-Brieux, Fr,2006 \\
\hline & $\mathrm{BVH} 25 \mathrm{~b}$ & 13 & - & - & 13 & 11 & 12 & 4 & 8 & 11 & 12 & $\begin{array}{l}\text { Human, Respiratory } \\
\text { tract }\end{array}$ & I & Saint-Brieux, Fr,2006 \\
\hline & $\mathrm{BVH} 26 \mathrm{a}$ & 13 & - & - & 13 & 11 & 12 & 4 & 8 & 11 & 12 & Human, Wound & । & Saint-Brieux, Fr,2006 \\
\hline & $\mathrm{BVH} 27 \mathrm{a}$ & 13 & - & - & 13 & 11 & 12 & 4 & 8 & 11 & 12 & Human, Wound & । & $\begin{array}{l}\text { Reunion Island, } \\
\text { Fr,2006 }\end{array}$ \\
\hline & $\mathrm{BVH} 28 \mathrm{a}$ & 13 & - & - & 13 & 11 & 12 & 4 & 8 & 11 & 12 & Human, Wound & । & $\begin{array}{l}\text { Reunion Island, } \\
\text { Fr,2006 }\end{array}$ \\
\hline & $\mathrm{BVH61}$ & 46 & 5 & $\mathrm{D}$ & 46 & 29 & 31 & 31 & 34 & 34 & 40 & Human, Stool & I & Antibes, Fr,2006 \\
\hline & $\mathrm{BVH71}$ & 54 & 5 & D & 46 & 29 & 31 & 31 & 26 & 34 & 40 & Human, Stool & ND & $\begin{array}{l}\text { Martinique Island, } \\
\mathrm{Fr}, \mathrm{ND}\end{array}$ \\
\hline & $\mathrm{BVH} 47$ & 33 & - & $\mathrm{D}$ & 33 & 29 & 31 & 31 & 26 & 16 & 31 & Human, Blood & 1 & Roubaix, Fr,2006 \\
\hline & ADV102 & 33 & - & $\mathrm{D}$ & 33 & 29 & 31 & 31 & 26 & 16 & 31 & Human, Stool & ND & Montpellier, Fr, 2008 \\
\hline & $\mathrm{BVH} 18$ & 10 & - & - & 10 & 9 & 10 & 10 & 7 & 9 & 9 & Human, Wound & I & $\begin{array}{l}\text { Villeneuve sur Lot, } \\
\mathrm{Fr}, 2006\end{array}$ \\
\hline & AK249 & 10 & - & - & 10 & 9 & 10 & 10 & 7 & 9 & 9 & $\begin{array}{l}\text { Environment, } \\
\text { Water lake }\end{array}$ & & Annecy, Fr, 1998 \\
\hline & ADV129 & 85 & 8 & $\mathrm{H}$ & 78 & 64 & 74 & 69 & 56 & 56 & 67 & Human, Stool & ND & Montpellier, Fr, 2009 \\
\hline
\end{tabular}

A. hydrophila subsp.

$\begin{array}{llll}85 & 8 & \mathrm{H} & 78\end{array}$ 
Table 1 Typing data and origin of Aeromonas strains analyzed in the study (Continued)

\begin{tabular}{|c|c|c|c|c|c|c|c|c|c|c|c|c|c|}
\hline ADV133 & 89 & 8 & $\mathrm{H}$ & 82 & 64 & 74 & 69 & 56 & 56 & 67 & Human, Wound & 1 & Montpellier, Fr, 2010 \\
\hline BVH 90 & 66 & 7 & G & 61 & 6 & 58 & 55 & 45 & 43 & 53 & Human, Stool & 1 & Dunkerque, Fr, 2006 \\
\hline AK236 & 106 & 7 & G & 61 & 6 & 58 & 55 & 45 & 68 & 53 & $\begin{array}{l}\text { Environment, } \\
\text { Water lake }\end{array}$ & - & Annecy, Fr, 1998 \\
\hline $\mathrm{BVH} 37$ & 25 & - & - & 25 & 21 & 23 & 24 & 20 & 19 & 23 & Human, Blood & 1 & $\begin{array}{l}\text { La Roche sur Yon, } \\
\text { Fr, } 2006\end{array}$ \\
\hline $\mathrm{BVH} 46$ & 25 & - & - & 25 & 21 & 23 & 24 & 20 & 19 & 23 & Human, Blood & 1 & Roubaix, Fr, 2006 \\
\hline $\mathrm{BVH} 56$ & 42 & 4 & $E$ & 42 & 36 & 40 & 24 & 32 & 6 & 23 & Human, Blood & 1 & Versailles, Fr, 2006 \\
\hline ADV101 & 74 & 4 & $E$ & 42 & 57 & 40 & 24 & 32 & 19 & 23 & Human, Stool & ND & Montpellier, Fr, 2008 \\
\hline $\begin{array}{l}\text { A. veronii bv. veronii } \\
\text { CECT } 4257^{\top}\end{array}$ & 143 & - & - & 131 & 114 & 125 & 120 & 11 & 19 & 110 & $\begin{array}{l}\text { Human, Respiratory } \\
\text { tract }\end{array}$ & 1 & Michigan, USA, NA \\
\hline A. veronii CCM 4360 & 143 & - & - & 131 & 114 & 125 & 120 & 11 & 19 & 110 & Human, Stool & 1 & $\begin{array}{l}\text { Connecticut, USA, } \\
1984\end{array}$ \\
\hline BVH6 & 4 & - & - & 4 & 4 & 4 & 4 & 4 & 4 & 4 & Human, Wound & 1 & Cahors, Fr, 2006 \\
\hline $\mathrm{BVH} 13$ & 6 & - & - & 6 & 6 & 6 & 6 & 4 & 6 & 6 & Human, Blood & 1 & Le Mans, Fr, 2006 \\
\hline $\mathrm{BVH} 26 \mathrm{~b}$ & 16 & - & - & 16 & 11 & 15 & 15 & 11 & 6 & 15 & Human, Wound & 1 & Saint-Brieux, Fr,2006 \\
\hline BVH31 & 19 & - & - & 19 & 16 & 18 & 18 & 14 & 16 & 18 & Human, Bile & । & La Rochelle, Fr, 2006 \\
\hline $\mathrm{BVH} 32$ & 20 & - & - & 20 & 17 & 19 & 19 & 15 & 17 & 19 & Human, Stool & C & La Rochelle, Fr, 2006 \\
\hline $\mathrm{BVH} 44$ & 31 & - & - & 31 & 27 & 29 & 30 & 20 & 24 & 29 & Human, Wound & 1 & Périgueux, Fr, 2006 \\
\hline BVH49 & 35 & - & - & 35 & 30 & 33 & 33 & 20 & 27 & 33 & Human, Stool & 1 & $\begin{array}{l}\text { Chalon sur Saone, } \\
\mathrm{Fr}, 2006\end{array}$ \\
\hline $\mathrm{BVH} 50$ & 36 & - & - & 36 & 31 & 34 & 34 & 28 & 6 & 34 & $\begin{array}{l}\text { Human, Respiratory } \\
\text { tract }\end{array}$ & 1 & $\begin{array}{l}\text { Chalon sur Saone, } \\
\text { Fr, } 2006\end{array}$ \\
\hline BVH53 & 39 & - & - & 39 & 34 & 37 & 37 & 31 & 30 & 29 & Human, Blood & 1 & Saint Denis, Fr, 2006 \\
\hline $\mathrm{BVH} 54$ & 40 & - & - & 40 & 35 & 38 & 38 & 4 & 31 & 37 & $\begin{array}{l}\text { Human, Respiratory } \\
\text { tract }\end{array}$ & 1 & Saint Denis, Fr, 2006 \\
\hline BVH59 & 44 & - & - & 44 & 37 & 41 & 39 & 33 & 19 & 38 & Human, Blood & 1 & Le Havre, Fr, 2006 \\
\hline $\mathrm{BVH} 60$ & 45 & - & - & 45 & 38 & 42 & 40 & 20 & 33 & 39 & Human, Stool & 1 & Antibes, Fr, 2006 \\
\hline $\mathrm{BVH73}$ & 56 & - & - & 39 & 43 & 50 & 37 & 40 & 38 & 47 & Human, Blood & ND & $\begin{array}{l}\text { Martinique Island, } \\
\mathrm{Fr}, \mathrm{NA}\end{array}$ \\
\hline $\mathrm{BVH77}$ & 58 & - & - & 54 & 45 & 52 & 50 & 41 & 19 & 49 & Human, Stool & C & $\begin{array}{l}\text { Aix en Provence, } \\
\text { Fr, } 2006\end{array}$ \\
\hline BVH79 & 59 & - & - & 55 & 46 & 53 & 51 & 4 & 19 & 37 & Human, Wound & 1 & $\begin{array}{l}\text { Aix en Provence, } \\
\mathrm{Fr}, 2006\end{array}$ \\
\hline $\mathrm{BVH} 80$ & 60 & - & - & 56 & 47 & 54 & 52 & 42 & 39 & 50 & Human, Stool & 1 & $\begin{array}{l}\text { Aix en Provence, } \\
\mathrm{Fr}, 2006\end{array}$ \\
\hline BVH95 & 69 & - & - & 64 & 53 & 61 & 58 & 20 & 46 & 55 & Human, Wound & 1 & $\begin{array}{l}\text { Bourg en Bresse, } \\
\text { Fr, } 2006\end{array}$ \\
\hline
\end{tabular}


Table 1 Typing data and origin of Aeromonas strains analyzed in the study (Continued)

\begin{tabular}{|c|c|c|c|c|c|c|c|c|c|c|c|c|c|}
\hline ADV103 & 75 & - & - & 68 & 29 & 66 & 62 & 26 & 34 & 59 & Human, Stool & ND & Montpellier, Fr, 2008 \\
\hline ADV109 & 78 & - & - & 71 & 60 & 69 & 65 & 53 & 51 & 61 & Human, Stool & ND & Montpellier, Fr, 2008 \\
\hline ADV1 19 & 80 & - & - & 73 & 61 & 70 & 66 & 53 & 52 & 62 & Human, Stool & ND & Montpellier, Fr, 2009 \\
\hline ADV125 & 83 & - & - & 76 & 63 & 72 & 68 & 54 & 54 & 65 & Human, Stool & ND & Montpellier, Fr, 2009 \\
\hline ADV127 & 84 & - & - & 77 & 11 & 73 & 68 & 55 & 55 & 66 & Human, Stool & ND & Montpellier, Fr, 2009 \\
\hline ADV130 & 86 & - & - & 79 & 11 & 75 & 70 & 33 & 17 & 68 & Human, Blood & ND & Montpellier, Fr, 2010 \\
\hline ADV131 & 87 & - & - & 80 & 65 & 76 & 71 & 11 & 19 & 69 & $\begin{array}{l}\text { Human, Respiratory } \\
\text { tract }\end{array}$ & ND & Montpellier, Fr, 2009 \\
\hline ADV135 & 90 & - & - & 83 & 67 & 78 & 73 & 57 & 58 & 23 & Human, Stool & ND & Montpellier, Fr, 2010 \\
\hline ADV137b & 91 & - & - & 84 & 68 & 79 & 74 & 58 & 59 & 71 & $\begin{array}{l}\text { Human, Respiratory } \\
\text { tract }\end{array}$ & ND & Montpellier, Fr, 2010 \\
\hline AK219 & 96 & - & - & 89 & 27 & 84 & 79 & 11 & 62 & 75 & $\begin{array}{l}\text { Environment, Waste } \\
\text { water treatment } \\
\text { lagoon }\end{array}$ & - & Montracol, Fr, 2006 \\
\hline AK222 & 97 & - & - & 90 & 73 & 85 & 80 & 62 & 19 & 76 & $\begin{array}{l}\text { Environment, Waste } \\
\text { water treatment } \\
\text { lagoon }\end{array}$ & - & Montracol, Fr, 2006 \\
\hline AK226 & 99 & - & - & 92 & 75 & 87 & 82 & 8 & 63 & 12 & $\begin{array}{l}\text { Environment, Waste } \\
\text { water treatment } \\
\text { lagoon }\end{array}$ & - & Montracol, Fr, 2006 \\
\hline AK227 & 100 & - & - & 93 & 76 & 88 & 83 & 34 & 64 & 29 & $\begin{array}{l}\text { Environment, Waste } \\
\text { water treatment } \\
\text { lagoon }\end{array}$ & - & Montracol, Fr, 2006 \\
\hline AK232 & 103 & - & - & 95 & 11 & 91 & 86 & 64 & 65 & 79 & $\begin{array}{l}\text { Environment, Waste } \\
\text { water treatment } \\
\text { lagoon }\end{array}$ & - & Montracol, Fr, 2006 \\
\hline AK237 & 107 & - & - & 98 & 80 & 94 & 51 & 67 & 19 & 82 & $\begin{array}{l}\text { Environment, } \\
\text { Water lake }\end{array}$ & - & Annecy, Fr, 1998 \\
\hline AK238 & 108 & - & - & 99 & 81 & 95 & 89 & 34 & 69 & 83 & $\begin{array}{l}\text { Environment, } \\
\text { Water lake }\end{array}$ & - & Annecy, Fr, 1998 \\
\hline AK239 & 109 & - & - & 100 & 82 & 73 & 68 & 55 & 6 & 84 & $\begin{array}{l}\text { Environment, } \\
\text { Water lake }\end{array}$ & - & Annecy, Fr, 1998 \\
\hline AK240 & 110 & - & - & 101 & 83 & 96 & 90 & 20 & 19 & 85 & $\begin{array}{l}\text { Environment, } \\
\text { Water lake }\end{array}$ & - & Annecy, Fr, 1998 \\
\hline AK241 & 111 & - & - & 102 & 84 & 97 & 91 & 20 & 56 & 86 & Non-human, Snail & I & Angers, Fr, 1995 \\
\hline AK242 & 112 & - & - & 25 & 85 & 98 & 92 & 68 & 19 & 23 & $\begin{array}{l}\text { Environment, } \\
\text { Water lake }\end{array}$ & - & Annecy, Fr, 1998 \\
\hline AK243 & 113 & - & - & 103 & 86 & 98 & 93 & 20 & 70 & 87 & Non-human, Snail & I & Angers, Fr, 1995 \\
\hline AK244 & 114 & - & - & 104 & 87 & 99 & 94 & 69 & 19 & 23 & $\begin{array}{l}\text { Environment, } \\
\text { Water lake }\end{array}$ & - & Annecy, Fr, 1998 \\
\hline
\end{tabular}


Table 1 Typing data and origin of Aeromonas strains analyzed in the study (Continued)

\begin{tabular}{|c|c|c|c|c|c|c|c|c|c|c|c|c|c|c|}
\hline & AK246 & 116 & - & - & 106 & 89 & 101 & 95 & 71 & 19 & 12 & Non-human, Snail & I & Angers, Fr, 1995 \\
\hline & AK247 & 117 & - & - & 107 & 90 & 102 & 96 & 58 & 72 & 89 & $\begin{array}{l}\text { Environment, } \\
\text { Water lake }\end{array}$ & - & Annecy, Fr, 1998 \\
\hline & AK248 & 118 & - & - & 108 & 91 & 103 & 97 & 34 & 73 & 90 & $\begin{array}{l}\text { Environment, } \\
\text { Water lake }\end{array}$ & - & Annecy, Fr, 1998 \\
\hline & AK250 & 119 & - & - & 109 & 27 & 83 & 98 & 11 & 19 & 75 & $\begin{array}{l}\text { Environment, } \\
\text { Water lake }\end{array}$ & - & Annecy, Fr, 1998 \\
\hline & AK251 & 120 & - & - & 110 & 92 & 104 & 99 & 20 & 19 & 91 & $\begin{array}{l}\text { Environment, } \\
\text { Water lake }\end{array}$ & - & Annecy, Fr, 1998 \\
\hline & $\begin{array}{l}\text { A. culicicola CIP } \\
107763^{\top}\end{array}$ & 124 & - & - & 114 & 96 & 108 & 4 & 33 & 77 & 95 & $\begin{array}{l}\text { Non-human, } \\
\text { Mosquito midgut }\end{array}$ & ND & Pune, India, 1997 \\
\hline & $\begin{array}{l}\text { A. ichthiosmia } \\
\text { CECT } 4486^{\top}\end{array}$ & 132 & - & - & 122 & 104 & 116 & 110 & 81 & 19 & 102 & $\begin{array}{l}\text { Environment, } \\
\text { Surface water }\end{array}$ & - & NA, Germany, 1986 \\
\hline & $\begin{array}{l}\text { A. veronii bv. } \\
\text { sobria LMG } 13067\end{array}$ & 144 & - & - & 132 & 115 & 126 & 121 & 8 & 93 & 12 & Non-human, Frog & 1 & Connecticut, USA, NA \\
\hline & A. veronii CECT 4902 & 161 & - & - & 148 & 131 & 143 & 136 & 103 & 108 & 126 & Environment, NA & - & NA, Germany, 1993 \\
\hline & A. veronii CECT 7059 & 164 & - & - & 151 & 133 & 145 & 138 & 33 & 109 & 39 & $\begin{array}{l}\text { Environment, } \\
\text { Drinking water }\end{array}$ & - & Zaragoza, Spain, 2002 \\
\hline \multirow[t]{14}{*}{ A. caviae $(n=34)$} & BVH16 & 9 & 1 & B & 9 & 8 & 9 & 9 & 3 & 8 & 8 & $\begin{array}{l}\text { Human, Respiratory } \\
\text { tract }\end{array}$ & C & Rambouillet, Fr, 2006 \\
\hline & BVH57 & 43 & 1 & B & 43 & 8 & 9 & 9 & 3 & 32 & 8 & Human, Blood & । & Versailles, Fr, 2006 \\
\hline & BVH63 & 47 & 6 & $\mathrm{~F}$ & 12 & 10 & 43 & 41 & 3 & 10 & 41 & Human, Blood & । & Macon, Fr, 2006 \\
\hline & BVH84 & 47 & 6 & $\mathrm{~F}$ & 12 & 10 & 43 & 41 & 3 & 10 & 41 & Human, Stool & । & $\begin{array}{l}\text { Aix en Provence, } \\
\text { Fr, } 2006\end{array}$ \\
\hline & BVH98 & 72 & - & $\mathrm{F}$ & 12 & 10 & 64 & 60 & 37 & 10 & 41 & Human, Wound & । & Brest, Fr, NA \\
\hline & ADV118 & 79 & 6 & $\mathrm{~F}$ & 72 & 10 & 43 & 8 & 3 & 10 & 41 & Human, Wound & । & Montpellier, Fr, 2009 \\
\hline & ADV121 & 81 & - & $\mathrm{F}$ & 74 & 10 & 43 & 8 & 3 & 3 & 63 & Human, Stool & ND & Montpellier, Fr, 2009 \\
\hline & $\mathrm{BVH} 48$ & 34 & 2 & C & 34 & 10 & 32 & 32 & 27 & 26 & 32 & Human, Vagina & C & $\begin{array}{l}\text { Monceau les mines, } \\
\mathrm{Fr}, 2006\end{array}$ \\
\hline & A. caviae CCUG 48892 & 175 & 2 & C & 34 & 10 & 32 & 32 & 27 & 3 & 32 & $\begin{array}{l}\text { Environment, } \\
\text { Water }\end{array}$ & & Uppsala, Sweden, 2004 \\
\hline & BVH19 & 11 & - & C & 11 & 10 & 3 & 11 & 3 & 10 & 10 & Human, Vagina & C & $\begin{array}{l}\text { Villeneuve sur Lot, } \\
\mathrm{Fr}, 2006\end{array}$ \\
\hline & BVH81 & 61 & - & C & 34 & 10 & 3 & 11 & 3 & 26 & 32 & Human, Stool & C & $\begin{array}{l}\text { Aix en Provence, } \\
\mathrm{Fr}, 2006\end{array}$ \\
\hline & BVH66 & 50 & - & C & 34 & 10 & 46 & 44 & 37 & 26 & 32 & Human, Wound & । & $\begin{array}{l}\text { Martinique Island, } \\
\mathrm{Fr}, 2006\end{array}$ \\
\hline & BVH55 & 41 & 3 & C & 41 & 10 & 39 & 12 & 3 & 26 & 32 & Human, Stool & 1 & Saint-Denis, Fr, 2006 \\
\hline & $\mathrm{BVH} 87$ & 64 & 3 & C & 59 & 10 & 39 & 12 & 3 & 26 & 32 & Human, Stool & । & Aix en Provence, Fr, 2006 \\
\hline
\end{tabular}


Table 1 Typing data and origin of Aeromonas strains analyzed in the study (Continued)

\begin{tabular}{|c|c|c|c|c|c|c|c|c|c|c|c|c|c|c|}
\hline & $\mathrm{BVH} 4$ & 3 & - & - & 3 & 3 & 3 & 3 & 3 & 3 & 3 & Human, Wound & 1 & Cahors, Fr, 2006 \\
\hline & BVH15 & 8 & - & - & 8 & 7 & 8 & 8 & 6 & 7 & 7 & Human, Blood & 1 & Grasse, Fr, 2006 \\
\hline & $\mathrm{BVH} 20$ & 12 & - & - & 12 & 10 & 11 & 12 & 3 & 8 & 11 & Human, Stool & 1 & Gonesse, Fr, 2006 \\
\hline & BVH51 & 37 & - & - & 37 & 32 & 35 & 35 & 29 & 28 & 35 & Human, Blood & I & Monaco, Fr, 2006 \\
\hline & BVH52 & 38 & - & - & 38 & 33 & 36 & 36 & 30 & 29 & 36 & Human, Blood & । & Monaco, Fr, 2006 \\
\hline & BVH67 & 51 & - & - & 49 & 32 & 47 & 45 & 3 & 8 & 35 & Human, Stool & ND & $\begin{array}{l}\text { Martinique Island, } \\
\mathrm{Fr}, \mathrm{NA}\end{array}$ \\
\hline & BVH85 & 62 & - & - & 57 & 48 & 55 & 11 & 3 & 40 & 8 & Human, Stool & I & $\begin{array}{l}\text { Aix en Provence, } \\
\mathrm{Fr}, 2006\end{array}$ \\
\hline & BVH86 & 63 & - & - & 58 & 49 & 56 & 53 & 43 & 41 & 51 & Human, Stool & $C$ & $\begin{array}{l}\text { Aix en Provence, } \\
\mathrm{Fr}, 2006\end{array}$ \\
\hline & BVH100 & 73 & - & - & 67 & 56 & 65 & 61 & 50 & 26 & 58 & Human, Wound & ND & Brest, Fr, ND \\
\hline & ADV106 & 77 & - & - & 70 & 59 & 68 & 64 & 52 & 50 & 35 & Human, Stool & ND & Montpellier, Fr, 2008 \\
\hline & ADV124 & 82 & - & - & 75 & 62 & 71 & 67 & 3 & 53 & 64 & Human, Stool & ND & Montpellier, Fr, 2009 \\
\hline & AK223 & 98 & - & - & 91 & 74 & 86 & 81 & 3 & 8 & 77 & $\begin{array}{l}\text { Environment, Waste } \\
\text { water treatment lagoon }\end{array}$ & - & Montracol, Fr, 2006 \\
\hline & AK229 & 101 & - & - & 34 & 77 & 89 & 84 & 37 & 3 & 78 & $\begin{array}{l}\text { Environment, Waste } \\
\text { water treatment } \\
\text { lagoon }\end{array}$ & - & Montracol, Fr, 2006 \\
\hline & AK231 & 102 & - & - & 94 & 78 & 90 & 85 & 63 & 26 & 32 & $\begin{array}{l}\text { Environment, Waste } \\
\text { water treatment lagoon }\end{array}$ & - & Montracol, Fr, 2006 \\
\hline & AK234 & 104 & - & - & 96 & 10 & 92 & 87 & 65 & 66 & 80 & $\begin{array}{l}\text { Environment, Waste } \\
\text { water treatment lagoon }\end{array}$ & - & Montracol, Fr, 2006 \\
\hline & AK245 & 115 & - & - & 105 & 88 & 100 & 11 & 70 & 71 & 88 & Environment, Water lake & - & Annecy, Fr, 1998 \\
\hline & A. caviae CECT $838^{\mathrm{T}}$ & 123 & - & - & 113 & 95 & 107 & 102 & 74 & 76 & 94 & Non-human, Guinea pig & । & NA, USA, NA \\
\hline & $\begin{array}{l}\text { A. hydrophila subsp. } \\
\text { anaerogenes CECT } 4221\end{array}$ & 128 & - & - & 118 & 100 & 112 & 106 & 50 & 3 & 99 & $\begin{array}{l}\text { Environment, } \\
\text { Used oil emulsion }\end{array}$ & - & NA, USA, NA \\
\hline & A. caviae CECT 4222 & 154 & - & - & 142 & 78 & 136 & 131 & 37 & 103 & 35 & Environment, Sewage & - & NA, NA, 1954 \\
\hline & A. caviae CECT 4226 & 155 & - & - & 118 & 125 & 137 & 11 & 50 & 3 & 120 & $\begin{array}{l}\text { Environment, } \\
\text { Used oil emulsion }\end{array}$ & - & NA, USA, 1953 \\
\hline \multirow[t]{3}{*}{ A. piscicola $(n=3)$} & A. piscicola LMG $24783^{\top}$ & 151 & & & 139 & 122 & 133 & 128 & 95 & 100 & 117 & $\begin{array}{l}\text { Non-human, } \\
\text { Salmon }\end{array}$ & । & Gallicia, Spain, 2005 \\
\hline & A. sobria CECT 4333 & 156 & 9 & J & 143 & 126 & 138 & 132 & 98 & 104 & 121 & $\begin{array}{l}\text { Non-human, } \\
\text { Diseased elver }\end{array}$ & । & Valencia, Spain, NA \\
\hline & $\begin{array}{l}\text { Aeromonas sp. } \\
\text { CECT } 5177\end{array}$ & 162 & 9 & J & 149 & 126 & 138 & 132 & 98 & 104 & 121 & $\begin{array}{l}\text { Environment, } \\
\text { Drinking water }\end{array}$ & - & Eeklo, Belgium, 1996 \\
\hline $\begin{array}{l}\text { A. salmonicida } \\
(\mathrm{n}=8)\end{array}$ & $\begin{array}{l}\text { A. salmonicida subsp. } \\
\text { achromogenes } \\
\text { CIP } 104001\end{array}$ & 136 & - & 1 & 126 & 107 & 120 & 114 & 85 & 86 & 94 & Non human, Trout & ND & Aberdeen, UK, 1963 \\
\hline
\end{tabular}


Table 1 Typing data and origin of Aeromonas strains analyzed in the study (Continued)

\begin{tabular}{|c|c|c|c|c|c|c|c|c|c|c|c|c|c|c|}
\hline & $\begin{array}{l}\text { A. salmonicida subsp. } \\
\text { masoucida } \\
\text { CIP } 103210\end{array}$ & 137 & - & 1 & 126 & 108 & 120 & 115 & 85 & 87 & 105 & $\begin{array}{l}\text { Non human, } \\
\text { Fish blood }\end{array}$ & 1 & NA, NA, 1969 \\
\hline & $\begin{array}{l}\text { A. salmonicida subsp. } \\
\text { smithia CIP } 104757\end{array}$ & 137 & - & 1 & 126 & 108 & 120 & 115 & 85 & 87 & 105 & $\begin{array}{l}\text { Non human, } \\
\text { Fish ulcer }\end{array}$ & 1 & NA, UK, NA \\
\hline & $\begin{array}{l}\text { A. salmonicida subsp. } \\
\text { salmonicida } \\
\text { CIP } 103209^{\top}\end{array}$ & 139 & - & । & 126 & 110 & 120 & 114 & 85 & 89 & 105 & $\begin{array}{l}\text { Non human, } \\
\text { Diseased salmon }\end{array}$ & 1 & Cletter river, UK, 1953 \\
\hline & BVH39 & 26 & - & - & 26 & 22 & 24 & 25 & 21 & 20 & 24 & Human, Wound & C & Vannes, Fr, 2006 \\
\hline & $\begin{array}{l}\text { A. salmonicida subsp. } \\
\text { pectinolytica } \\
\text { CIP } 107036\end{array}$ & 138 & - & - & 127 & 109 & 121 & 116 & 86 & 88 & 106 & $\begin{array}{l}\text { Environment, } \\
\text { River water }\end{array}$ & & $\begin{array}{l}\text { Buenos Aires, } \\
\text { Argentina, NA }\end{array}$ \\
\hline & A. salmonicida CCM 1150 & 168 & - & - & 155 & 136 & 149 & 142 & 108 & 112 & 131 & Non human,Fish & ND & $\begin{array}{l}\text { NA, Czech Republic, } \\
1961\end{array}$ \\
\hline & A. salmonicida CCM 1275 & 170 & - & - & 157 & 138 & 151 & 144 & 110 & 114 & 133 & Fish & ND & $\begin{array}{l}\text { NA, Czech Republic, } \\
1961\end{array}$ \\
\hline \multirow{3}{*}{$\begin{array}{l}\text { A. allosaccharophila } \\
(\mathrm{n}=3)\end{array}$} & BVH88 & 65 & - & - & 60 & 50 & 57 & 54 & 44 & 42 & 52 & Human, Blood & 1 & Dunkerque, Fr, 2006 \\
\hline & $\begin{array}{l}\text { A. allosaccharophila } \\
\text { CECT } 4199^{\top}\end{array}$ & 121 & - & - & 111 & 93 & 105 & 100 & 72 & 74 & 92 & Non-human, Fish & । & Valencia, Spain, 1991 \\
\hline & A. sobria CECT 4053 & 153 & - & - & 141 & 124 & 135 & 130 & 97 & 102 & 119 & $\begin{array}{l}\text { Environment, } \\
\text { Activated sludge }\end{array}$ & & $\begin{array}{l}\text { Stockholm, Sweden, } \\
1978\end{array}$ \\
\hline \multirow[t]{5}{*}{ A. sobria $(\mathrm{n}=5)$} & A. sobria CECT $4245^{\top}$ & 141 & - & - & 129 & 112 & 123 & 118 & 88 & 91 & 108 & Non-human, Fish & ND & NA, Fr, 1974 \\
\hline & $\begin{array}{l}\text { Aeromonas sp. } \\
\text { CECT } 4816\end{array}$ & 157 & - & - & 144 & 127 & 139 & 133 & 99 & 105 & 122 & Non-human, Fish & ND & NA, NA, 1993 \\
\hline & $\begin{array}{l}\text { Aeromonas sp. } \\
\text { CECT } 4817\end{array}$ & 158 & - & - & 145 & 128 & 140 & 134 & 100 & 106 & 123 & Non-human, Fish & ND & NA, NA, 1993 \\
\hline & $\begin{array}{l}\text { Aeromonas sp. } \\
\text { CECT } 4818\end{array}$ & 159 & - & - & 146 & 129 & 141 & 135 & 101 & 107 & 124 & Non-human, Fish & ND & NA, NA, 1993 \\
\hline & A. sobria CECT 4821 & 160 & - & - & 147 & 130 & 142 & 118 & 102 & 91 & 125 & Non-human, Fish & ND & NA, NA, 1993 \\
\hline \multirow[t]{6}{*}{ A. aquariorum $(n=8)$} & $\mathrm{BVH} 28 \mathrm{~b}$ & 17 & - & - & 17 & 14 & 16 & 16 & 12 & 14 & 16 & Human, Wound & 1 & $\begin{array}{l}\text { Reunion Island, } \\
\mathrm{Fr}, 2006\end{array}$ \\
\hline & $\mathrm{BVH} 43$ & 30 & - & - & 30 & 26 & 28 & 29 & 25 & 23 & 28 & Human, Wound & 1 & Périgueux, Fr, 2006 \\
\hline & BVH65 & 49 & - & - & 48 & 39 & 45 & 43 & 36 & 36 & 43 & Human, Blood & 1 & $\begin{array}{l}\text { Martinique Island, } \\
\mathrm{Fr}, 2006\end{array}$ \\
\hline & BVH68 & 52 & - & - & 50 & 40 & 48 & 46 & 12 & 23 & 44 & Human, NA & ND & $\begin{array}{l}\text { Martinique Island, } \\
\mathrm{Fr}, \mathrm{ND}\end{array}$ \\
\hline & $\mathrm{BVH70}$ & 53 & - & - & 51 & 41 & 28 & 47 & 38 & 37 & 45 & Human, NA & ND & $\begin{array}{l}\text { Martinique Island, } \\
\mathrm{Fr}, \mathrm{ND}\end{array}$ \\
\hline & ADV132 & 88 & - & - & 81 & 66 & 77 & 72 & 25 & 57 & 70 & Human, Wound & 1 & Montpellier, Fr, 2010 \\
\hline
\end{tabular}


Table 1 Typing data and origin of Aeromonas strains analyzed in the study (Continued)

\begin{tabular}{|c|c|c|c|c|c|c|c|c|c|c|c|c|c|c|}
\hline & $\begin{array}{l}\text { A. hydrophila subsp. } \\
\text { dhakensis CIP } 107500\end{array}$ & 129 & - & - & 119 & 101 & 113 & 107 & 78 & 23 & 100 & Human, Stool & 1 & NA, Bangladesh, NA \\
\hline & $\begin{array}{l}\text { A. aquariorum } \\
\text { CECT } 7289^{\top}\end{array}$ & 145 & - & - & 133 & 116 & 127 & 122 & 89 & 94 & 111 & Non-human, Fish & ND & NA, Portugal, 2003 \\
\hline \multirow[t]{6}{*}{ A. media $(n=6)$} & $\mathrm{BVH} 40$ & 27 & - & - & 27 & 23 & 25 & 26 & 22 & 21 & 25 & Human, Stool & C & Vannes, Fr, 2006 \\
\hline & AK202 & 92 & - & - & 85 & 69 & 80 & 75 & 59 & 60 & 72 & Non-human, Snail & । & Angers, Fr, 1995 \\
\hline & AK211 & 94 & - & - & 87 & 71 & 82 & 77 & 61 & 60 & 73 & Non-human, Snail & । & Angers, Fr, 1995 \\
\hline & A. media CECT $4232^{\top}$ & 134 & - & - & 124 & 71 & 118 & 112 & 83 & 84 & 97 & $\begin{array}{l}\text { Environment, Fish } \\
\text { farm effluent water }\end{array}$ & - & $N A, U K, N A$ \\
\hline & Aeromonas sp. CECT 7111 & 167 & - & - & 154 & 71 & 148 & 141 & 107 & 60 & 130 & Non-human, Oyster & - & Barcelona, Spain, NA \\
\hline & A. media CCM 4242 & 173 & - & - & 159 & 141 & 154 & 147 & 59 & 117 & 136 & $\begin{array}{l}\text { Environment, } \\
\text { River water }\end{array}$ & - & $\begin{array}{l}\text { NA, Czech Republic, } \\
1991\end{array}$ \\
\hline \multirow[t]{3}{*}{ A. tecta $(n=3)$} & A. tecta CECT $7082^{\top}$ & 146 & - & - & 134 & 117 & 128 & 123 & 90 & 95 & 112 & Human, Stool & ND & Ticino, Switzerland, NA \\
\hline & Aeromonas sp. CECT 7081 & 165 & - & - & 152 & 134 & 146 & 139 & 105 & 110 & 128 & Non-human, Fish & ND & $\begin{array}{l}\text { Ticino, Switzerland, } \\
1983\end{array}$ \\
\hline & Aeromonas sp. CECT 7083 & 166 & - & - & 153 & 135 & 147 & 140 & 106 & 111 & 129 & Environment, Tap water & - & $\begin{array}{l}\text { Ticino, Switzerland, } \\
1993\end{array}$ \\
\hline A. jandaei & BVH92 & 67 & - & - & 62 & 51 & 59 & 56 & 46 & 44 & 54 & Human, Urine & 1 & Toulouse, Fr, 2006 \\
\hline$(n=2)$ & A. jandaei CECT $4228^{\top}$ & 133 & - & - & 123 & 105 & 117 & 111 & 82 & 83 & 103 & Human, Stool & ND & Oregon, USA, 1980 \\
\hline A. enteropelogenes & $\begin{array}{l}\text { A. enteropelogenes } \\
\text { CECT } 4487^{\top}\end{array}$ & 126 & - & - & 116 & 98 & 110 & 104 & 76 & 79 & 97 & Human, Stool & ND & NA, India, NA \\
\hline A. trota & A. trota CECT $4255^{\top}$ & 142 & - & - & 130 & 113 & 124 & 119 & 76 & 92 & 109 & Human, Stool & ND & Varasani, India, NA \\
\hline A. bestiarum & A. bestiarum CECT $4227^{\top}$ & 122 & - & - & 112 & 94 & 106 & 101 & 73 & 75 & 93 & Non-human, Fish & ND & NA, Fr, 1974 \\
\hline A. encheleia & A. encheleia CECT $4342^{\top}$ & 125 & - & - & 115 & 97 & 109 & 103 & 75 & 78 & 96 & Non-human, Fish & । & Valencia, Spain, 1987 \\
\hline HG11 & HG11 CECT 4253 & 147 & - & - & 135 & 118 & 129 & 124 & 91 & 96 & 113 & Human, Wound & । & New Zealand, 1983 \\
\hline A. eucrenophila & $\begin{array}{l}\text { A. eucrenophila } \\
\text { CECT } 4224^{\top}\end{array}$ & 127 & - & - & 117 & 99 & 111 & 105 & 77 & 80 & 98 & $\begin{array}{l}\text { Non-human, } \\
\text { Freshwater fish }\end{array}$ & ND & $N A, N A, N A$ \\
\hline A. fluvialis & $\begin{array}{l}\text { A. fluvialis } \\
\text { LMG } 24681^{\top}\end{array}$ & 149 & - & - & 137 & 120 & 131 & 126 & 93 & 98 & 115 & $\begin{array}{l}\text { Environmental, } \\
\text { River water }\end{array}$ & - & Girona, Spain, NA \\
\hline A. popoffii & A. popoffi CIP $105493^{\top}$ & 135 & - & - & 125 & 106 & 119 & 113 & 84 & 85 & 104 & Environmental, Water & - & $\begin{array}{l}\text { Oelegem, Belgium, } \\
1993\end{array}$ \\
\hline A. sanarellii & A. sanarellii LMG $24682^{\top}$ & 152 & - & - & 140 & 123 & 134 & 129 & 96 & 101 & 118 & Human, Wound & । & NA, Taïwan, 2000 \\
\hline A. schubertii & A. schubertii CECT $4240^{\top}$ & 140 & - & - & 128 & 111 & 122 & 117 & 87 & 90 & 107 & Human, Wound & । & Texas, USA, 1981 \\
\hline A. diversa & HG13 CECT $4254^{\top}$ & 148 & - & - & 136 & 119 & 130 & 125 & 92 & 97 & 114 & Human, Wound & 1 & Louisiana, USA, NA \\
\hline A. taiwanensis & $\begin{array}{l}\text { A. taiwanensis } \\
\text { LMG } 24683^{\top}\end{array}$ & 150 & - & - & 138 & 121 & 132 & 127 & 94 & 99 & 116 & Human, Wound & । & NA, Taïwan, 2000 \\
\hline Unknown taxon & $\begin{array}{l}\text { A. bestiarum } \\
\text { CCM } 1271\end{array}$ & 169 & - & - & 156 & 137 & 150 & 143 & 109 & 113 & 132 & Non-human, Gold fish & ND & $N A, N A, N A$ \\
\hline
\end{tabular}


Table 1 Typing data and origin of Aeromonas strains analyzed in the study (Continued)

\begin{tabular}{|c|c|c|c|c|c|c|c|c|c|c|c|c|c|c|}
\hline A. bivalvium & A. bivalvium CECT $7113^{\top}$ & - & - & - & 161 & 142 & 155 & - & 112 & 119 & 138 & Non-human, Cockles & - & Barcelona, Spain, 1997 \\
\hline A. molluscorum & $\begin{array}{l}\text { A. molluscorum CIP } \\
108876^{\top}\end{array}$ & - & - & - & - & 143 & 156 & - & 113 & 120 & 139 & $\begin{array}{l}\text { Non-human, } \\
\text { Wedge-shells }\end{array}$ & - & Barcelona, Spain, 1997 \\
\hline A. simiae & $\begin{array}{l}\text { A. simiae CIP } \\
107798^{\top}\end{array}$ & - & - & - & 162 & 144 & 157 & - & 114 & 121 & 140 & $\begin{array}{l}\text { Non-human, } \\
\text { Healthy monkey }\end{array}$ & - & NA, Mauritus, 1999 \\
\hline A. rivuli & $\begin{array}{l}\text { A. rivuli DSM } \\
22539^{\top}\end{array}$ & - & - & - & - & 145 & 158 & 149 & 115 & 122 & 141 & $\begin{array}{l}\text { Environment, } \\
\text { Karst hardwater creek }\end{array}$ & - & $\begin{array}{l}\text { Westerhöfer Bach, } \\
\text { Germany, NA }\end{array}$ \\
\hline
\end{tabular}

For each strain, the table presents the results of the multilocus phylogenetic analysis (MLPA); the result of the multilocus sequence analysis (strain ST number and belonging to a clonal complex); the allelic number for each of the 7 genes; and the characteristics of strain isolation (origin, colonizing or infecting status, spatio-temporal origin).

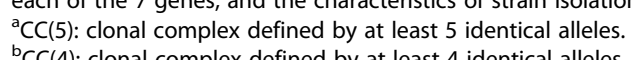

ND: not determined; NA: not available; -: not applicable.

ND: not determined; NA: not available, -: not applicable.

All bacteremia originated from the gut [17]. 
primers used in this study are described in Table 2. Genomic DNA was obtained using the Aquapure DNA extraction kit (EpiCentre, Madison, WI). PCR was carried out in a $50 \mu \mathrm{L}$ reaction mixture containing 200 $\mathrm{nM}$ of each primer (Sigma Genosys), $200 \mu \mathrm{M}$ of each deoxynucleoside triphosphate (dNTP) (Euromedex, Mundolsheim, France), $2 \mathrm{mM} \mathrm{MgCl}_{2}$, and $2.5 \mathrm{U}$ of Taq DNA polymerase (Promega, Madison, WI) in the appropriate reaction buffer and $50 \mathrm{ng}$ of genomic DNA as the template. The amplification conditions were as follows: initial denaturation for $4 \mathrm{~min}$ at $94^{\circ} \mathrm{C}$, followed by $35 \mathrm{amp}$ lification cycles as indicated in Table 2 and a final extension step at $72^{\circ} \mathrm{C}$ for $10 \mathrm{~min}$. zip $A$ amplification required specific conditions for some $A$. caviae and $A$. media isolates included in this study, such as a $4 \mathrm{mM} \mathrm{MgCl}_{2}$ concentration and a primer hybridization temperature of $50^{\circ} \mathrm{C}$ (A. caviae).

The PCR products and a molecular weight marker (phage phiX DNA digested with HaeIII, New England BioLabs) were separated in $1.5 \%$ agarose gels in $0.5 \mathrm{X}$ TBE buffer. The products were then sequenced using forward amplification primers (Table 2) in an ABI 3730XL automatic sequencer (Beckman Coulter Genomics, United Kingdom).

\section{Phylogenetic analysis}

Gene sequences were codon aligned using the ClustalW application within the Bioedit Sequence Alignment Editor [19]. The sizes of the codon-aligned sequences that were used for further analyses are indicated in Table 2. Phylogenetic analyses were performed for each of the 7 gene sequences and for a manually concatenated sequence. Gaps in concatenated sequences were deleted with Bioedit. The sequences were converted in Phylip format using the ReadSeq online program (http://searchlauncher. bcm.tmc.edu/seq-util/readseq.html). A distance-based phylogenetic tree was reconstructed using the neighbor-joining method implemented in Neighbor from the PHYLIP package v3.66 [20]. Distances were calculated using FastDist. The K2P substitution model was selected for the analysis plus a gamma parameter fixed to 2. Distance bootstrap support was calculated after 1000 reiterations. The analysis was performed online on the site www.phylogeny.fr. For the maximum likelihood (ML) method-based phylogeny, evolutionary distance was analyzed with the PhyML v3.0 program [21] using GTR, with a gamma distribution parameter estimated from the dataset and invariant sites as a substitution model. This substitution model was determined to be the most appropriate by ModelTest [22]. ML bootstrap support was calculated after 100 reiterations.

\section{Multilocus sequence analysis}

For each locus, each allele was assigned a distinct arbitrary number using a nonredundant database program available at http://www.pubmlst.org. The combination of allele numbers for each isolate defined the sequence type (ST).

Allele profiles were analyzed using eBURST v3 software [23] to determine the clonal complexes (CCs) defined as sets of related strains that share at least 5 identical alleles at the 7 loci. A complementary eBURST analysis was conducted to determine the CCs sharing at least 4 identical alleles at the 7 loci.

The program LIAN 3.5 [24], available at http://www. pubmlst.org, was used to calculate the standardized index of association (sIA) to test the null hypothesis of linkage disequilibrium, the mean genetic diversity $(\mathrm{H})$ and the genetic diversity at each locus (h). The number of synonymous (dS) and non-synonymous (dN) substitutions per site was determined from codon-aligned sequences using Sequence Type Analysis and Recombinational Tests Version 2 (START2) software [25]. Other genetic analyses, including the determination of allele and allelic profile frequencies, mol\% $\mathrm{G}+\mathrm{C}$ content and polymorphic site numbering, were also carried out using START2 software. A distance matrix in nexus format was generated from the set of allelic profiles and then used for decomposition analyses with SplitsTree 4.0 software [26]. Recombination events were detected from the aligned ST concatenated sequences using the RDP v3.44 [27] software package with the following parameters: general (linear sequence, highest $P$ value of 0.05 , Bonferroni correction), RDP (no reference, window size of 8 polymorphic sites, $0-100 \%$ sequence identity range), GENECONV (scan triplets, G-scale of 1), Bootscan (window size of $200 \mathrm{bp}$, step size of $20 \mathrm{bp}, 70 \%$ cutoff, F84 model, 100 bootstrap replicates, binomial $P$ value), MAxChi (scan triplets, fraction of variable sites per window set to 0.1), CHIMAERA (scan triplets, fraction of variable sites per window set to 0.1 ) and Siscan (window of $200 \mathrm{bp}$, step size of $20 \mathrm{bp}$, use 1/2/3 variable positions, nearest outlier for the $4^{\text {th }}$ sequence, $1000 P$ value permutations, 100 scan permutations).

\section{Other statistics}

All qualitative variables with the exception of the sIA were compared using a Chi-squared test or the Fisher's exact test where appropriate; a $P$ value $\leq 0.05$ was considered to reflect significance. All computations were performed using $\mathrm{R}$ project software (http://www.r-project.org).

\section{Nucleotide sequence accession numbers}

The nucleotide sequences determined in this study were deposited in the GenBank database with the following accession numbers: JN828973 - JN829166 (dnaK), JN829167 - JN829361 ( $g l t A)$, JN829362 - JN829556 ( gyrB), JN829557 - JN829748 (radA), JN829749 - JN829943 (rpoB), JN829944 - JN830138 (tsf), and JN830139 - JN830333 (zipA). 


\section{Table 2 Primers and amplification conditions}

\begin{tabular}{|c|c|c|c|c|c|c|c|c|}
\hline Locus & Function & Putative gene product & Gene size $^{a}(b p)$ & $\begin{array}{c}\text { Locus } \\
\text { position }^{\text {a }}(\mathrm{bp})\end{array}$ & Primer $^{b}$ & Primer sequence $5^{\prime}-3^{\prime}$ & $\begin{array}{l}\text { Sequence length amplified } \\
\text { (alignment length) (bp) }\end{array}$ & Amplification conditions \\
\hline dnak & Stress response & $\begin{array}{l}\text { Heat shock } 70 \\
\text { kDa protein (HSP 70) }\end{array}$ & 1,928 & 3357741 & $\begin{array}{l}\text { DnaK-F } \\
\text { DnaK-R }\end{array}$ & $\begin{array}{l}\text { ATGAAGAAGACCGCCGAAG } \\
\text { TGCAGCACGTGAATGGTC }\end{array}$ & $816(816)$ & $\begin{array}{l}45 \mathrm{~s} \text { at } 94^{\circ} \mathrm{C} ; \\
45 \mathrm{~s} \text { at } 63^{\circ} \mathrm{C} ; \\
45 \mathrm{~s} \text { at } 72^{\circ} \mathrm{C}\end{array}$ \\
\hline$g / t A$ & Glycolytic pathway & $\begin{array}{l}\text { Type II citrate } \\
\text { synthase }\end{array}$ & 1,286 & 2098162 & $\begin{array}{l}\text { GltA-F } \\
\text { GltA-R }\end{array}$ & $\begin{array}{l}\text { TTCCGTCTGCTCTCCAAGAT } \\
\text { GCAGCGGATCCTTGATCT }\end{array}$ & $462(462)$ & $\begin{array}{l}45 \mathrm{~s} \text { at } 94^{\circ} \mathrm{C} ; \\
45 \mathrm{~s} \text { at } 63^{\circ} \mathrm{C} ; \\
45 \mathrm{~s} \text { at } 72^{\circ} \mathrm{C}\end{array}$ \\
\hline gyrB & Replication & Subunit DNA gyrase & 2,411 & 3883 & $\begin{array}{l}\text { GyrB3F } \\
\text { GyrB14R }\end{array}$ & (Yáñez, 2003) & 783-786(792) & $\begin{array}{l}45 \mathrm{~s} \text { at } 94^{\circ} \mathrm{C} ; \\
45 \mathrm{~s} \text { at } 65^{\circ} \mathrm{C} ; \\
45 \mathrm{~s} \text { at } 72^{\circ} \mathrm{C}\end{array}$ \\
\hline $\operatorname{rad} A$ & DNA repair & $\begin{array}{l}\text { DNA repair protein, } \\
\text { RadA }\end{array}$ & 1,364 & 4108941 & $\begin{array}{l}\text { RadA-F } \\
\text { RadA-R }\end{array}$ & $\begin{array}{l}\text { ATGCATCACCTGGATGGAGT } \\
\text { TGCCTATGTTTGACCGAATG }\end{array}$ & $405-414(420)$ & $\begin{array}{l}45 \mathrm{~s} \text { at } 94^{\circ} \mathrm{C} ; \\
45 \mathrm{~s} \text { at } 60^{\circ} \mathrm{C} ; \\
45 \mathrm{~s} \text { at } 72^{\circ} \mathrm{C}\end{array}$ \\
\hline$r p o B$ & Transcription & $\begin{array}{l}\text { DNA-directed RNA } \\
\text { polymerase } \beta \text { subunit }\end{array}$ & 4,091 & 4466890 & $\begin{array}{l}\text { Pasrpob-L } \\
\text { Rpob-R }\end{array}$ & (Korczak, 2004) & $426(426)$ & $\begin{array}{l}45 \mathrm{~s} \text { at } 94^{\circ} \mathrm{C} ; \\
45 \mathrm{~s} \text { at } 65^{\circ} \mathrm{C} ; \\
45 \mathrm{~s} \text { at } 72^{\circ} \mathrm{C}\end{array}$ \\
\hline tsf & Protein translation & Elongation factor Ts & 881 & 1272267 & $\begin{array}{l}\text { Tsf-F } \\
\text { Tsf-R }\end{array}$ & $\begin{array}{l}\text { CGCTGGCATGATGGATTG } \\
\text { GATGCCTTCGCCCACTTC }\end{array}$ & 702(702) & $\begin{array}{l}45 \mathrm{~s} \text { at } 94^{\circ} \mathrm{C} ; \\
45 \mathrm{~s} \text { at } 65^{\circ} \mathrm{C} ; \\
45 \mathrm{~s} \text { at } 72^{\circ} \mathrm{C}\end{array}$ \\
\hline zipA & Cell division & $\begin{array}{l}\text { Cell division } \\
\text { protein ZipA }\end{array}$ & 1,112 & 1337458 & $\begin{array}{l}\text { ZipA-F } \\
\text { ZipA-R }\end{array}$ & $\begin{array}{l}\text { TTGCGTTACATCTTGGTTGC } \\
\text { ATCCGGTACGGATTGAAGGT }\end{array}$ & $429-507(537)$ & $\begin{array}{l}1 \mathrm{~min} \text { at } 94^{\circ} \mathrm{C} ; \\
45 \mathrm{~s} \text { at } 55^{\circ} \mathrm{C}_{i} \\
1 \mathrm{~min} \text { at } 72^{\circ} \mathrm{C}\end{array}$ \\
\hline
\end{tabular}

${ }^{a}$ Gene start codon position on the circular chromosome sequence of $A$. hydrophila subsp. hydrophila ATCC $7966^{\top}$ (accession number NC_008570).

${ }^{b}{ }_{F}$, forward primer; $R$, reverse primer.

'Some strains belonging to $A$. caviae and $A$. media clades were amplified using a $4 \mathrm{mM} \mathrm{MgCl}_{2}$ concentration and a primer hybridization temperature of $50^{\circ} \mathrm{C}(A$. caviae). 


\section{Results}

\section{MLSA scheme design}

Seven genes encoding housekeeping proteins involved in replication $(\mathrm{gyr} B)$, transcription $(r p o B)$, DNA repair $(\operatorname{radA})$, the glycolytic pathway $(g l t A)$, stress responses $(d n a K)$, protein translation (tsf), and cell division (zipA) were selected (Table 2). They were dispersed on the chromosomes of both complete genome sequences used for gene selection to minimize the influence of physical links between loci (Table 2). Partial sequences of the 7 genes were obtained for 191 out of the 195 strains. We failed to obtain a $\mathrm{radA}$ partial sequence for A. bivalvium CECT $7113^{\mathrm{T}}, A$. molluscorum CIP $108876^{\mathrm{T}}$, and A. simiae CIP $107798^{\mathrm{T}}$ and a dnaK partial sequence for A. molluscorum CIP $108876^{\mathrm{T}}$ and A. rivuli DSM $22539^{\mathrm{T}}$. Therefore, these type strains were not included in the multilocus analysis. An alternative 5-gene scheme excluding $\mathrm{radA}$ and $d n a K$ was used to perform a complete phylotaxonomic analysis in aeromonads. The sizes of the sequences at each locus are given in Table 2; the 7-locus concatemere length was $4155 \mathrm{nt}$.

\section{Phylotaxonomics}

The population structure was inferred from multilocus phylogenetic analysis (MLPA) following reconstruction of the distance and ML trees from the concatenated sequences (alignment length of $3993 \mathrm{nt})$. The ML concatenated tree is shown in Figure 1, and the differences in the relative branching order between methods are also highlighted in Figure 1. The Aeromonas population was organized into 11 clades, which included 2 to 71 strains, with three major clades being observed (bootstrap values $\geq 90$ ). The largest clade was comprised of 71 isolates, including 46 human, 5 animal and 20 environmental isolates, among which 4 were reference strains and three were type strains: A. culicicola CIP $107763^{\mathrm{T}}$, A. ichthiosmia CECT $4486^{\mathrm{T}}, A$. veronii biovar sobria LMG 13067 and $A$. veronii biovar veronii CECT $4257^{\mathrm{T}}$; this was designated the $A$. veronii clade (Figure 1, Table 1). The two other major clades included 35 and 34 strains, respectively. They were referred to as the A. hydrophila clade (including strains A. hydrophila subsp. hydrophila CECT $839^{\mathrm{T}}, A$. hydrophila subsp. ranae CIP 107985 and 33 other isolates) and the A. caviae clade (including A. caviae CECT $838^{\mathrm{T}}$, A. hydrophila subsp. anaerogenes CECT 4221 and 32 other isolates), respectively. Each of these clades contained strains from various sources, i.e., 25 human, 7 animal and 3 environmental strains in the A. hydrophila cluster and 24 human, 9 environmental and 1 animal isolate in the A. caviae cluster (Figure 1, Table 1). The remaining strains were distributed among eight minor clades (bootstrap values $\geq 90$ ), and are presented in Table 1 and Figure 1. The relative branching order among clades remains uncertain for most nodes (Figure 1). The clades displayed a mean sequence divergence of $2.5 \%$, but the A. media clade displayed higher genetic polymorphism than the other clades (5.8\%). None of the isolates included in this study grouped with the type strains $A$. bestiarum, A. diversa, A. encheleia, A. enteropelogenes, A. eucrenophila, A. fluvialis, A. popoffi, A. sanarellii, A. schubertii, A. taiwanensis, and A. trota, or with the representative strain of hybridization group 11. Finally, strain CCM 1271 formed an independent phylogenetic branch that was clearly differentiated from related known species, particularly from $A$. bestiarum, the species name under which the strain is referenced in the Czech Collection of Microorganisms (Figure 1). A phylogenetic tree reconstructed for all the strains included in this study using a concatenated sequence of the 5 genes obtained for all of the strains also showed strain CCM 1271 to be unrelated to A. bivalvium CECT $7113^{\mathrm{T}}$, A. molluscorum CIP $108876^{\mathrm{T}}$, A. simiae CIP $107798^{\mathrm{T}}$ and A. rivuli DSM $22539^{\mathrm{T}}$ (see Additional file 1: Figure S1).

The multilocus sequence-based phylogeny supported the current taxonomy of the genus. In addition, both the high level of concatenated sequence divergence observed in the $A$. media cluster and the comparison of the subtree topology for clusters including closely related known species, such as $A$. eucrenophila- $A$. encheleia- $A$. tecta, suggested that the $A$. media clade may constitute a polyphyletic cluster containing taxa that have yet to be described. Strain CCM 1271, showing a clearly segregated phylogenetic position in the MLPA, also likely represents an unknown Aeromonas taxon.

\section{Genetic diversity}

The number of different alleles for the 7 loci varied from 111 (rpoB) to 160 (dnaK) (Table 3). This significant variation $\left(P\right.$ value $\left.=10^{-9}\right)$ suggested distinct mutation rates among the loci. The equivalent mol\% $\mathrm{G}+\mathrm{C}$ content ranged from $55.8(t s f)$ to $62.6 \%(\operatorname{radA})$ for all loci with the exception of zipA, which exhibited a lower mol\% G + C content of $52.4 \%$. The mean genetic diversity among strains was high for the whole genus, and of the 3 main clades, A. caviae displayed the lowest genetic diversity (h) for all genes (Table 3). The rate of polymorphic sites varied significantly between the $A$. caviae, A. hydrophila and $A$. veronii clades for all loci except for rpoB, with $A$. caviae being the clade that showed the lowest number of polymorphic sites for all loci (Table 3). The rates of non-synonymous versus synonymous substitutions $(\mathrm{dN} /$ dS ratio) were rather low, except for zipA, indicating that among the 7 loci, only one locus had been subjected to strong positive selective pressure (Table 3).

\section{Multilocus sequence typing, genomic relationships and origin of the strains}

The multilocus sequence dataset for the 191 strains contained 175 sequence types (STs), 164 (93.7\%) of which were identified only once. ST diversity was 0.92 per 


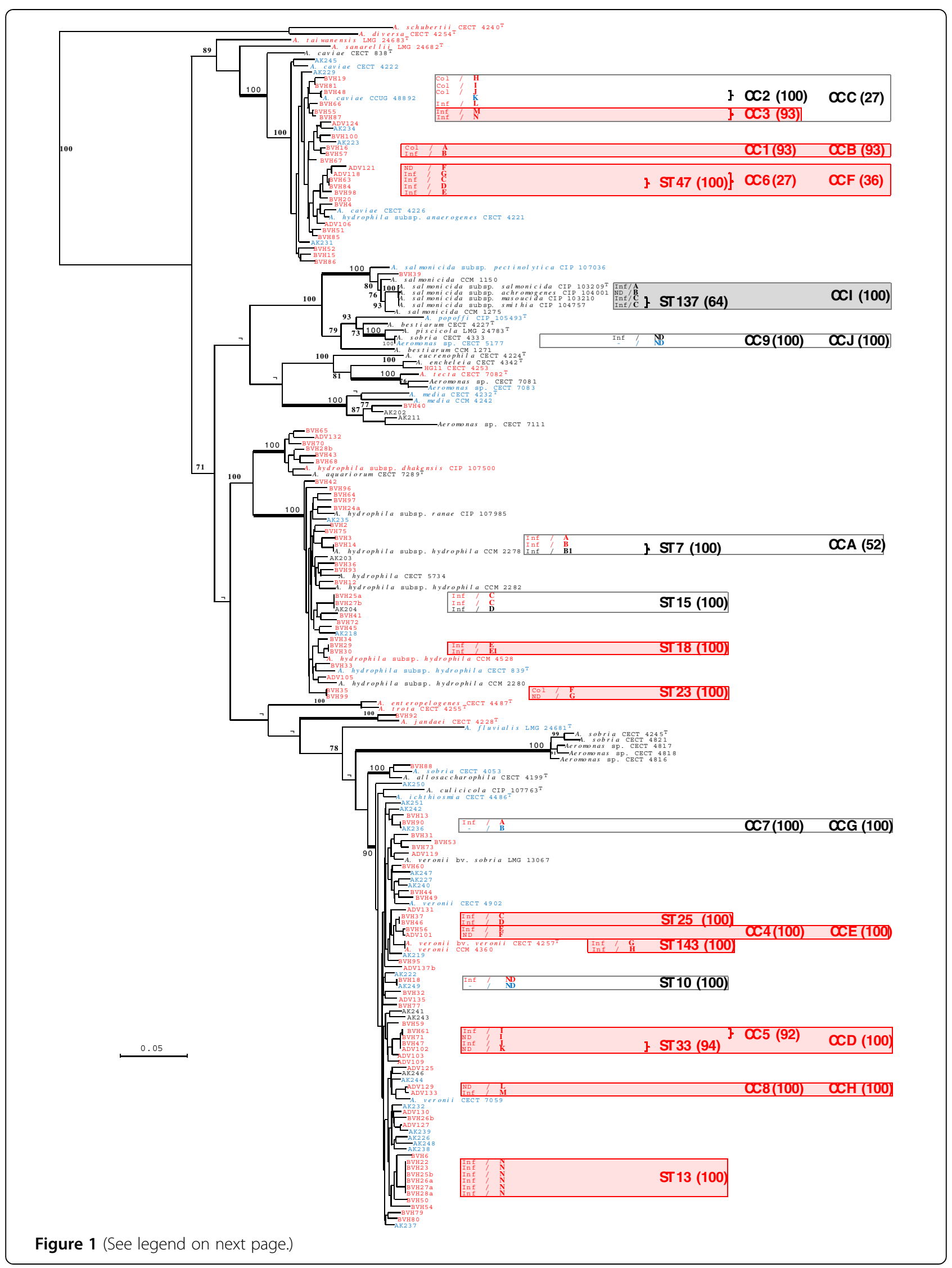




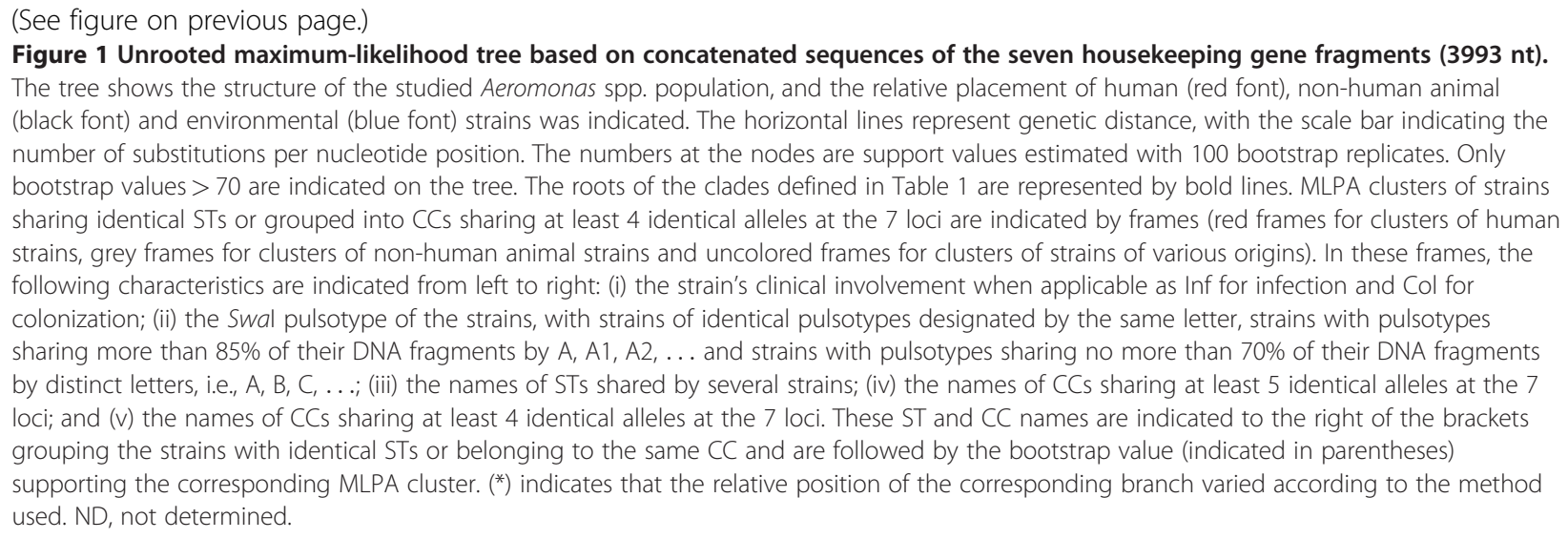

strain for the genus Aeromonas, confirming its exceptionally high level of population diversity, which was also observed in the A. caviae, A. hydrophila and A. veronii clades, which exhibited $0.97,0.86$ and 0.87 ST per strain, respectively. The largest ST group included 6 strains of the $A$. veronii clade. A total of 10 other STs were shared by a maximum of 3 strains (Table 1, Figure 1 ).

The clustering of STs in CCs sharing at least 5 identical alleles at the 7 loci revealed $9 \mathrm{CCs}$, which grouped a maximum of 3 strains. These CCs corresponded to MLPA clades supported by high bootstrap values $\geq 92 \%$, except for CC " 6 " (Figure 1, Table 1). Using a less stringent definition of CCs (4 identical allele at the 7 loci) did not significantly change the population clustering, confirming that the high genetic diversity of the population was equally expressed at each locus (Table 1, Figure 1 and 2).

Links among strains sharing the same ST and strains grouped into CCs were further investigated by comparing their geographic origins and isolation dates and using PFGE. The genomic macro-restriction digest with the endonuclease SwaI produced PFGE patterns that comprised of an average of 18 bands suitable for strain comparison (data not shown). The strains grouped within each of these clusters showed distinct pulsotypes and/or were of distinct geographic origin and, in some cases, had been isolated over a long time period. For example, ST7 included strains BVH14 and CCM 2278, sharing more than $85 \%$ of their DNA fragments in the PFGE analysis, which were isolated in France in 2006 and in California in 1963, respectively (Table 1, Figure 1). Of particular note, the largest ST found in this study, ST13, included 6 strains with identical pulsotypes, despite being isolated in 2006 from distant sites (e.g., La Réunion Island in the Indian ocean and 2 distant regions in mainland France). Finally, we observed that the type strains of $A$. salmonicida subsp. masoucida and $A$. salmonicida subsp. smithia purchased from the Collection of the Institut Pasteur showed identical STs and pulsotypes; this questionable result should be considered with caution until a further control analysis is performed in strains ordered from another collection.

Comparison of the overall diversity observed according to the origin of the strains within the 3 main clades showed that the number of STs per strain differed significantly between the groups of clinical and environmental isolates ( 0.875 and 1 , respectively; $P$ value $=0.036)$. This difference also reached the level of significance among the $A$. veronii group $(P$ value $=0.049)$. A few robust clusters of strains were shown to group isolates from the same host origin, which primarily grouped strains of human origin (Figure 1, Table 1). Out of the 43 geographically and/or genomically unrelated strains belonging to the 3 main clades included in either identical ST groups or in clonal complexes, only 3 originated from the environment, and 2 were involved in animal diseases (Table 1 and Figure 1). This difference in the distribution of environmental/animal and human clinical strains was statistically significant $\left(P\right.$ value $\left.=5.10^{-4}\right)$ for the 3 main clades and for the $A$. veronii $(P$ value $=0.02)$ and $A$. caviae $(P$ value $=0.05)$ clades.

Finally, a non-random distribution of strains was observed among the different CCs according to their site of isolation and/or colonizing/pathogenic status. CC " $C$ " grouped 3 out of the 5 non-pathogenic, colonizing $A$. caviae strains in the dataset, and this rate was significantly different from that of the non-pathogenic $A$. caviae strains found outside of the CC $(P$ value $=0.04)$ (Table 1, Figure 1). In contrast, some other clusters included strains involved only in infectious processes (Table 1, Figure 1). Finally, the A. veronii ST13 cluster appeared to be associated with a particular type of disease, i.e., wound infection. Indeed, 5 out of the $12 \mathrm{~A}$. veronii strains in the dataset involved in wound infection were grouped into this cluster, representing a frequency that was significantly different from the rest of the $A$. veronii population $(P$ value $=0.0001)$. 
Table 3 Sequence analysis for the seven loci of the MLSA scheme

\begin{tabular}{|c|c|c|c|c|c|c|c|}
\hline Locus & Genus or Clade & $\begin{array}{l}\text { No. of alleles } \\
\text { (\% per clade) }\end{array}$ & $\begin{array}{l}\text { No. (\%) of } \\
\text { polymorphic } \\
\text { sites }\end{array}$ & $\begin{array}{l}\text { Genetic diversity } \\
\text { (h) }\end{array}$ & $\begin{array}{l}\text { No. of non-synonymous } \\
\text { codons }\end{array}$ & $\mathrm{dN} / \mathrm{dS}$ ratio & $\mathrm{G}+\mathrm{C} \%^{\mathrm{b}}$ \\
\hline \multirow[t]{5}{*}{ dnak } & Genus ( $n=191)$ & 160 & $273(33.5)$ & 0.9970 & 56 & 0.27 & 58.8 \\
\hline & A. caviae $(n=34)$ & $26(76.5)$ & $70(8.6)$ & 0.9697 & 3 & 0.006 & 60.1 \\
\hline & A. hydrophila $(\mathrm{n}=35)$ & $29(82.9)$ & $87(10.7)$ & 0.9882 & 0 & 0 & 59.5 \\
\hline & A. veronii $(n=71)$ & $57(81.4)$ & $119(14.6)$ & 0.9901 & 0 & 0 & 57.8 \\
\hline & $P$ value & NS & $6.10^{-8}$ & - & 0.036 & - & - \\
\hline \multirow[t]{5}{*}{$g / t A$} & Genus ( $n=191)$ & 141 & $160(30.5)$ & 0.9900 & 21 & 0.048 & 61.5 \\
\hline & A. caviae $(n=34)$ & $18(52.9)$ & $28(6.1)$ & 0.8324 & 4 & 0.089 & 61.8 \\
\hline & A. hydrophila $(\mathrm{n}=35)$ & $26(74.3)$ & $41(8.9)$ & 0.9714 & 1 & 0.006 & 62.5 \\
\hline & A. veronii $(n=71)$ & $50(75.7)$ & $70(15.1)$ & 0.9735 & 1 & 0.002 & 60.8 \\
\hline & $P$ value & NS & $6.10^{-7}$ & - & NS & - & - \\
\hline \multirow[t]{5}{*}{ gyrB } & Genus ( $n=191$ ) & 154 & $278(35.1)$ & 0.9966 & 39 & 0.035 & 59.1 \\
\hline & A. caviae $(n=34)$ & $26(76.5)$ & $58(7.3)$ & 0.9786 & 2 & 0.004 & 60.7 \\
\hline & A. hydrophila $(\mathrm{n}=35)$ & $28(80.0)$ & $92(11.6)$ & 0.9885 & 3 & 0.005 & 59.6 \\
\hline & A. veronii $(\mathrm{n}=71)$ & $55(82.9)$ & $137(17.3)$ & 0.9884 & 7 & 0.012 & 58 \\
\hline & $P$ value & NS & $10^{-10}$ & - & NS & - & - \\
\hline \multirow[t]{5}{*}{$\operatorname{rad} A$} & Genus ( $n=191)$ & 148 & $194(46.6)$ & 0.9955 & 30 & 0.061 & 62.6 \\
\hline & A. caviae $(n=34)$ & $23(67.6)$ & $28(6.7)$ & 0.9661 & 1 & 0.007 & 63.4 \\
\hline & A. hydrophila $(\mathrm{n}=35)$ & $28(80.0)$ & $61(14.5)$ & 0.9832 & 5 & 0.029 & 64.6 \\
\hline & A. veronii $(n=71)$ & $50(71.4)$ & $66(15.7)$ & 0.9801 & 6 & 0.009 & 61.1 \\
\hline & $P$ value & NS & $10^{-14}$ & - & NS & - & - \\
\hline \multirow[t]{5}{*}{$r p o B$} & Genus ( $n=191$ ) & 111 & $98(23.0)$ & 0.9846 & 6 & 0.004 & 57 \\
\hline & A. caviae $(n=34)$ & $13(38.2)$ & $18(4.2)$ & 0.7683 & 1 & 0.013 & 58.7 \\
\hline & A. hydrophila $(\mathrm{n}=35)$ & 24 (68.6) & $24(5.6)$ & 0.9681 & 0 & 0 & 56.3 \\
\hline & A. veronii $(n=71)$ & $31(44.3)$ & $25(5.9)$ & 0.9528 & 0 & 0 & 56.4 \\
\hline & $P$ value & 0.02 & 0.31 & - & NS & - & - \\
\hline \multirow[t]{5}{*}{ tsf } & Genus ( $n=191$ ) & 118 & $177(27.1)$ & 0.9844 & 30 & 0.068 & 55.8 \\
\hline & A. caviae $(n=34)$ & $16(47.1)$ & $16(2.3)$ & 0.9073 & 1 & 0.015 & 56.5 \\
\hline & A. hydrophila $(\mathrm{n}=35)$ & $21(60.0)$ & $24(3.4)$ & 0.9445 & 1 & 0.008 & 55.9 \\
\hline & A. veronii $(\mathrm{n}=71)$ & $37(52.9)$ & $79(11.8)$ & 0.9288 & 9 & 0.032 & 55.3 \\
\hline & $P$ value & NS & $3.10^{-5}$ & - & 0.004 & - & - \\
\hline \multirow[t]{5}{*}{ zipA } & Genus ( $n=191)$ & 137 & $380(70.8)$ & 0.9929 & 130 & 0.333 & 52.4 \\
\hline & A. caviae $(n=34)$ & $20(58.8)$ & $98(18.3)$ & 0.9358 & 31 & 0.276 & 52.9 \\
\hline & A. hydrophila $(\mathrm{n}=35)$ & $25(71.4)$ & $31(5.8)$ & 0.9697 & 6 & 0.071 & 53.6 \\
\hline & A. veronii $(\mathrm{n}=71)$ & $46(66.2)$ & $50(9.3)$ & 0.9718 & 12 & 0.158 & 51.3 \\
\hline & $P$ value & NS & $3.10^{-5}$ & - & $10^{-5}$ & - & - \\
\hline
\end{tabular}

${ }^{\mathrm{a}}$ Mean genetic diversity $(\mathrm{H})$ among strains for the whole genus: $0.9916 \pm 0.0020$ and for the main 3 A. hydrophila, A. caviae, A. veronii clades: $0.9724 \pm 0.0055$, $0.9083 \pm 0.0301$ and $0.9694 \pm 0.0082$, respectively.

${ }^{\mathrm{b}}$ Mean G+C\% values for the 7 loci: $58.15 \%$ for the 191 Aeromonas spp. strains of this study, 59.2\% for A. caviae clade, $58.9 \%$ A. hydrophila clade and $57.2 \%$ A. veronii clade.

NS: not significant, dN/dS: rate of non-synonymous versus synonymous substitutions.

-: not determined.

$P$ value indicates result of statistical tests for data comparison between clades.

Recombination events in the aeromonad population The sIA value was 0.30 at the genus level, ranged from 0.15 to 0.42 at the clade level and was significantly different from 0 , indicating the existence of significant linkage disequilibrium, showing that the studied Aeromonas population was not panmictic but clonal. Events of 
recombination among the clonal population were then analyzed via RDP, decomposition analysis and phylogenetic incongruence.

Considering the recombination events detected using at least 4 methods of the RDP software, 14 types of recombination events leading to 166 recombinant sequences were detected among the population and are detailed in an additional table (Additional file 2: Table S2). All but two loci $(\operatorname{rad} A$ and $r p o B)$ were affected by recombination events that occurred in 89 STs (50.9\%). dnaK and $g y r B$ were the most affected loci ( 4 events each, 75 and 13 recombinant sequences, respectively), followed by tsf and zipA ( 3 events each, 73 and 5 recombinant sequences, respectively) and gltA (1 event and 3 recombinant sequences) (data not shown). One to four types of significant recombination events occurred in most clades, except for the $A$. hydrophila, A. piscicola and A. tecta clades and the A. fluvialis type strain and strain CCM 1271. Five events could not be significantly linked to parental sequences, suggesting the occurrence of transfer from strains that are not represented in our collection.

Recombination was also investigated for the 3 main clades via split decomposition in the concatenated sequences (Additional file 3: Figure S3 a-c). Most of the STs were not affected by recombination, and the trees showed a limited parallelogram formation, notably including A. hydrophila STs (Additional file 3: Figure S3 b). Split decomposition detected more recombination events within the CCs, particularly for STs in the A. caviae clade (Additional file 3: Figure S3 a).

Distance and ML trees were reconstructed for each of the 7 genes and compared to the concatenated sequencebased trees. For all genes and phylogenetic methods, single locus phylogenies (SLPA) displayed lower bootstrap values than MLPA trees (data not shown). Moreover, differences in branching order were observed in SLPA, suggesting the occurrence of recombination events (data not shown). In detail, phylogenetic discordance was observed for 11 strains based on single-gene phylogenetic analysis: all of these strains grouped in a robust cluster that was different from the cluster defined based on the 6 other genes or the concatenated sequence (shown in bold text in Table 1). Identical alleles were observed in strains belonging to different MLPA clusters, i.e., gyrB allele 83, common to the two environmental strains $A$. veronii strain AK250 and $A$. hydrophila strain AK218; zipA allele 97, common to the $A$. media and $A$. enteropelogenes type strains; and zip $A$ allele 94, which was identical in the $A$. caviae type strain and $A$. salmonicida strain CIP104001 (Table 1). In addition, strain BVH53 belonged to the A. veronii clade in the MLPA, while it was robustly grouped with the $A$. jandaei type strain in the gyrB-based phylogeny (bootstrap value of $100 \%$ in both the ML and distance-based trees) (data not shown). Similarly, among the isolated strains, the $A$. fluvialis type strain showed a divergent phylogenetic position between the $g l t A$-based tree, where it robustly grouped with the $A$. schubertii type strain, and other genebased phylogenies or the MLPA. Finally, strain BVH39 grouped within the $A$. salmonicida clade in the multilocus tree, while it was excluded from the corresponding clade defined in the $d n a K$-based tree. These phylogenetic incongruities revealed a total of 12 recombination events $(0.9 \%$ of the sequences), which occurred in 11 strains (4, 3 and 4 strains of human, animal and environmental origin, respectively) (5.8\% of the total strains) and concerned 5 out of the 7 genes addressed in our MLSA scheme, i.e., dnaK (1 strain), gltA (1 strain), gyrB (4 strains), tsf (3 strains), and zipA (3 strains) (Table 1). Multilocus phylogenetic trees reconstructed excluding the strains subjected to recombination showed increased bootstrap values for the $A$. veronii clade (90 to $100 \%$ ) as well as for most interclade nodes, confirming that recombination distorted the MLPA (data not shown). Despite its relatively low frequency of occurrence in the genus Aeromonas, recombination may account, at least in part, for some controversial taxonomic issues. For example, strain CCM 1271 is closely related to $A$. bestiarum in the gyrB-based phylogenetic tree (data not shown), whereas it is clearly individualized from this species in the MLPA.

\section{Discussion}

In this study, we investigated the genetic diversity and population structure linked with strain origin using MLSA. This work, based on a large collection of clinical isolates, i) estimated high genetic diversity, recombination rates and horizontal gene transfer in Aeromonas species, which together highlighted the mode of evolution in this group; ii) showed small clusters of strains associated with human infection; and iii) provided phylotaxonomic data that helped clarify the confusing taxonomy of the genus Aeromonas in relation to other works $[15,16,28]$. These results are further discussed below.

\section{A MLSA scheme for studying Aeromonas spp. population structure}

This was the $3^{\text {rd }}$ multilocus scheme proposed for studying Aeromonas spp. in 2011 [15,16]. These three studies analyzed different populations of aeromonads with different set of genes and different objectives. The $1^{\text {st }}$ MLSA scheme was developed for analyzing Aeromonas phylogeny and attempting to resolve the taxonomic controversies within this genus [16]. The $2^{\text {nd }}$ was developed to achieve precise strain genotyping and phylogenetic analysis of outbreak traceability and genetic diversity and was based on strains isolated from fish, crustaceans and mollusks [15]. The MLSA that we have presented here improved the understanding of human aeromonosis by 
addressing a large population that included both clinical and environmental strains from diverse geographic sources. The overall collection represented different lifestyles encountered in the genus: free living or associated with humans or cold-blooded animals. The clinical strain collection was representative of the French epidemiology because it resulted from a systematic prospective nationwide record and was associated with well-documented clinical reports [17]. The size of the collection was increased by including strains from various collections, most of which came from animal and environmental sources, so that the overall collection studied herein totaled 195 strains, which is a greater number compared to the two other MLSA studies on Aeromonas [15,16]. Our MLSA scheme was suitable for analysis of the whole genus Aeromonas, with the exception of four species: $A$. bivalvium, A. molluscorum, A. simiae and A. rivuli, for which only 6 genes could be analyzed. This MLPA allowed structuring the population into 3 main clades, designated $A$. veronii, $A$. hydrophila and $A$. caviae, because they contained the type strains of these species. Despite the fact that the number of isolates in the main clades was high compared to the study by Martino et al. [15] and similar to other studies [e.g., 29], the number of strains in some clades remained rather limited (e.g., $A$. caviae: 34 strains), and our results should be confirmed in a larger population. For this purpose, the population results and MLSA scheme have been deposited in a public database (PubMLST: http://pubmlst.org/software/ database/bigdb/) [30]. Nevertheless, our results provided interesting insight into the genetic diversity and structure of the Aeromonas population encountered in clinical infections as well as the mode of evolution of this population.

The MLSA scheme presented here included 7 housekeeping genes, which were sporadically distributed among the genome and, thus, can reasonably be assumed to not be associated with mobile genetic elements. The 7 genes encoded components of different metabolic pathways and were characterized by different mutation rates and low positive selective pressure, indicating predominantly neutral evolution of the loci. In our MLSA scheme, we propose the use of 4 genes not previously included in the 2 other MLSA schemes (dnaK, $\operatorname{radA}, t s f$ and $z i p A)$ for inferring the Aeromonas population structure. Despite its markedly lower mol\% G $+\mathrm{C}$ content compared to other loci and to the mean value for the genus Aeromonas inferred from the A. hydrophila ATCC $7966^{\mathrm{T}}$, A. salmonicida A449, and A. caviae Ae398 genomes (approximately 61.5\%) and the $A$. veronii B565 genome (58.8\%) [2,31-33], $z i p A$ was included in the MLSA scheme because the zipAbased phylogenetic tree was mostly congruent with those obtained for other genes (data not shown). This suggested that this gene likely originated from a distant genus through lateral genetic transfer, though it was likely acquired by a common ancestor of the population studied. Altogether, the 3 available multilocus schemes for Aeromonas indicated 16 distinct genes, i.e., atpD, dnaJ, dnaK, dnaX, gltA, groL, gyrA, gyrB, metG, ppsA, $\operatorname{rad} A, \operatorname{rec} A, \operatorname{rpoB}$, $\operatorname{rpoD}$, tsf, and zipA, that will offer the possibility of performing accurate analyses in Aeromonas.

\section{Mode of evolution}

Both the genetic and ST diversity per strain were observed to be exceptionally high in the genus Aeromonas and were much higher than observed for many other environmental bacteria $[9,11,34]$. Although strains from countries other than France only represented approximately $25 \%$ of the total strains of our dataset, the high level of genetic diversity observed validated the nonredundancy and representativeness of our population. Given that some geographically distant strains were very closely related (e.g., BVH 14 and CCM 2278, (Figure 1 and Table 1)), the global genetic diversity of this group may be reflected in that of a rather small sampling population, as observed in other reports on water-living species with high genetic diversities, such as Pseudomonas aeruginosa [35]. However, further analysis will be required to confirm this hypothesis. High diversity was observed in the 3 main A. caviae, A. hydrophila and $A$. veronii clades; however, the genetic characteristics and population structure of the $A$. caviae clade were outstanding. Compared to the other two mains groups, the A. caviae clade showed a lower genetic diversity, indicated both by its genetic diversity (h) and lower number of polymorphic sites. However, $A$. caviae exhibited the highest $\mathrm{dN} / \mathrm{dS}$ ratio for 4 genes. These results suggested that $A$. caviae strains have experienced less genetic variation, but when such variations have occurred, the mutations have more often been non-synonymous. Positive selective pressure or genetic hitchhiking is unlikely to explain this phenomenon. In this context, the occurrence of deleterious mutations linked to demographic effects experienced by the population represents a hypothesis that can explain the genetic particularities of $A$. caviae. The high genetic diversity in the genus, as observed by other researchers as well $[15,36]$ reflects the behavior of aeromonads as water-living bacteria. In fact, Aeromonas represent an outstanding example of generalist bacteria displaying genetic and genomic traits associated with this lifestyle and their ability to adapt to diverse niches, i.e., a relatively large genome $(4.7 \mathrm{Mb})$ [2], high genetic diversity, significant rate of horizontal gene transfer of housekeeping genes (5.8\% in our population), a significant number of ribosomal operons that are sometimes heterogeneous and submitted to cross-over events [37-39], genomic and phenotypic plasticity [2] and a great ability to adapt to new niches. All of this diversity corresponded to 
structuring in terms of complexes of species rather than species sensu stricto [40]. The wide range of genetic repertoires included in these complexes of species may constitute a potential reservoir for the emergence of future specialists via a speciation process related to selective pressure within a narrow niche. The complex and confusing systematics of the genus Aeromonas may result, at least in part, from the structure in species complexes in which speciation is progressing locally. For example, the species status of A. allosaccharophila, a clade closely related to $A$. veronii, has long been controversial, and evidence indicating whether this represents a definitive species has varied according to the methods used and the housekeeping genes analyzed [16,28,41-45]. If speciation is currently in progress for A. allosaccharophila, it could explain these controversial data, as highlighted by Silver [28] or as observed in other genera (e.g., the Burkholderia cepacia complex [46]). In contrast, $A$. salmonicida could represent an example of a fishadapted species subjected to some costs of specialization (e.g., being non-motile, having the ability to growth at $25^{\circ} \mathrm{C}$ but not at $37^{\circ} \mathrm{C}$ ) [33]. In this study, $A$. caviae appeared to have exceptional genetics compared to $A$. veronii or $A$. hydrophila. The hypothesis of a population bottleneck related to adaptation to a specialized niche, such as the gut, which is a more frequent niche for $A$. caviae compared to other aeromonads, should be emphasized. In fact, compared to $A$. veronii and $A$. hydrophila, A. caviae is preferentially found in the gut, as highlighted by the higher frequencies of gastroenteritis and bacteremia infections originating from the gut [17] and the higher density of $A$. caviae in wastewater inflows than outflows $[47,48]$

The diversity was significantly lower among the clinical strains than the environmental strains, which is compatible with the hypothesis that some clinical strains may correspond to niche-adapted subpopulations. Robust MLPA clusters of strains with identical STs or belonging to CCs were identified among the population, mainly among the 3 main clades this study. Each of these clusters included a limited number of strains (2 to 6 strains) that were further shown to be unrelated based on epidemiological data and/or PFGE results, and 52 out of the 191 fully analyzed strains (27.2\%) were involved in these clusters. Twelve clusters grouped strains from a unique host, i.e., a fish-associated subset within $A$. salmonicida and 11 human-associated subsets within the $A$. veronii $(\mathrm{n}=6)$, A. caviae $(\mathrm{n}=3)$ and A. hydrophila $(\mathrm{n}=2)$ clades. Nine of these subsets included only disease-associated strains. Notably, all of the A. veronii human-associated clusters were disease associated. Among these clusters, ST13, which was shared by 6 strains of human origin and was mainly recovered during wound infections, may reflect a host (niche)-adapted pathogenic cluster among the $A$. veronii clade, which was otherwise characterized by high genetic diversity. The striking, unique PFGE pattern and ST may reflect the adaptation of this cluster to a niche in which genetic and genomic variability is not permitted due to strong constraints. However, because of the small number of strains included in these clusters, an increased number of strains should be studied to confirm whether specific lineages or CCs are more likely to contain clinical isolates or be associated with a specific illness.

The present study showed a relatively low frequency of recombination events compared to previous studies $[15,28]$. This result may originate in the differences between these studies in the genes evaluated and the population sampling strategies employed. The population sample studied by Martino et al. differed significantly from ours, as most of their isolates were obtained from fish, crustaceans or mollusks [15]. Silver et al. deliberately included a very small number of isolates $(n=12)$ of host-associated strains (e.g., only strains isolated from leeches, human wounds or human feces), which may constitute a recruitment bias because these strains may be host adapted [28]. Interestingly, the recombination events encountered in our study were predominantly observed within clonal complexes (e.g., CC "D", grouping A. veronii strains recovered during human diarrhea episodes), which supported the previous hypothesis of the study by Silver et al. [28].

\section{Taxonomic considerations}

MLPA may be helpful for identification purposes. Indeed, strains that have previously rarely been reported in the literature were recognized among the study population, among which an $A$. jandaei isolate from a human urinary tract infection and an A. allosaccharophila strain recovered during human bacteremia were particularly remarkable. Moreover, MLPA may allow the correct identification of strains deposited in strain collections under erroneous or incomplete nomenclature, as observed for A. sobria CECT 4333 and Aeromonas sp. CECT 5177, which most likely belong to the $A$. piscicola species.

Multilocus sequence-based phylogeny supported recent taxonomic changes in the genus Aeromonas. First, several recently characterized species were clearly individualized in the 7 gene-based phylogenetic trees, such as A. taiwanensis, A. sanarellii and A. fluvialis [49,50]. The proposal of $A$. diversa, including Aeromonas sp. HG13, referred to as Aeromonas group 501, as a distinct species from A. schubertii was supported in the MLPA by the clearly individualized phylogenetic positions observed for these two species [51]. Moreover, several taxonomic reappraisals were confirmed by our approach, as observed and discussed in the MLPA study by MartinezMurcia et al. [16,52]. In addition, evidence previously 
suggesting that $A$. hydrophila subsp. anaerogenes and $A$. caviae are conspecific was confirmed here by the $A$. hydrophila subsp. anaerogenes strain CECT 4221 that was found to belong to the $A$. caviae clade [53]. All of these observations showed that the MLSA scheme appeared to be a strongly informative tool that should be included within the methods used for polyphasic taxonomic analysis in the genus Aeromonas. Thus, this MLSA scheme may provide additional arguments regarding controversial issues recently reviewed by Janda \& Abbott [1]. A. ichthiosmia, which is considered to be a later synonym of $A$. veronii [42], clearly grouped in the A. veronii clade. A. encheleia showed a low level of genetic divergence at the 7 loci and grouped in a tight and robust clade with HG11, providing additional arguments for their unification. A. allosaccharophila, whose existence is still controversial, occupies a robust position that is closely related, but external to the $A$. veronii clade, in favor of the separation of the two taxa. However, the taxonomic level of the new taxon, if proposed, still has to be defined due to conflicting DNA-DNA hybridization values compared to the $A$. veronii type strain according to the study considered [42,54]. Finally, the $A$. caviae type strain occupies a position external to those of other members of the $A$. caviae clade in the MLPA-based tree. This observation warrants further investigation due to the taxonomic value of the MLSA scheme demonstrated here. Of note, only 2 genes $(g y r B$ and $r p o B)$ from $A$. sharmana, a species that was shown not to belong to the genus Aeromonas and is awaiting reassignment, could be amplified using the primers employed in this study $[55,56]$.

\section{Conclusions}

Evolution in the genus Aeromonas has involved the combined effects of mutations and recombination events, resulting in an exceptionally high genetic diversity. We propose a hypothetical mode of evolution in aeromonads based on global organization into a complex of species, with local emergence of more specialized clones. This specialization in process is suggested by the co-existence of i) specialized species sensu stricto, such as A. salmonicida, ii) clades showing genetic traits associated with speciation and adaptation to a specific niche, such as $A$. caviae, and iii) diverse subsets of strains that may be host adapted and/or "disease specialized". The MLSA scheme developed herein in a large and diverse population of strains helped shed light on the unclear relationships among Aeromonas strains and aeromonosis. However, certain clades and the host- and/or disease-associated subsets of strains detected in this study included a limited number of strains. As a consequence, additional studies are required to increase the size of the analyzed population and to confirm these results. Further work including a virulence analysis focusing on human clinical clusters is also needed. Finally, the MLSA scheme proposed here appeared to be useful for taxonomic studies in the genus Aeromonas.

\section{Additional files}

\begin{abstract}
Additional file 1: Figure S1. Unrooted maximum-likelihood tree based on concatenated sequences of five housekeeping gene fragments ( $g / t A$, gyrB, rpoB, tsf, zipA, $2724 \mathrm{nt}$ ). The horizontal lines indicate genetic distance, with the scale bar indicating the number of substitutions per nucleotide position. The numbers at the nodes are support values estimated with 100 bootstrap replicates. Only bootstrap values $>70$ are shown on the tree. The clades defined in Table 1 are indicated with brackets at the top right of the figure. Only type strains and reference strains are represented in the tree.

Additional file 2: Table S2. Recombination event types and recombinant sequences.

Additional file 3: Figure S3. SplitsTree decomposition analyses of the MLSA data for strains belonging to the A. caviae (a), A. hydrophila (b) and A. veronii (c) clades. The distance matrix was obtained from the allelic profiles of the sequence types (ST). A network-like graph indicates recombination events. Star-like radiation from the central point indicates an absence of recombination. The names of eBURST clonal complexes (CCs), as defined in the text and in Table 1, are indicated near the corresponding STs. The number of strains sharing an identical ST is indicated below the ST number in brackets. Type strain STs are indicated by dots.
\end{abstract}

\section{Acknowledgments and funding}

We are particularly indebted to the microbiology laboratory team of the Montpellier, France, academic hospital for providing some clinical isolates. This work was supported by the Association des Biologistes de l'Ouest, by the Laboratoire de Diagnostic Bactériologique de l'Ecole Nationale Vétérinaire de Lyon and by ADEREMPHA (Association pour la Recherche et le Développement en Microbiologique \& Pharmacie).

We would like to thank all members of the colBVH study group who participated in this study: F. Carmagnol (Cannes), E. Chachaty (Institut Gustave Roussy), C. Alba-Sauviat (Chaumont), C. Auvray (Charleville-Mézières), D. Barraud (Gonesse), Z. Benseddik (Chartres), A. Bertrou (Carcassone), F. Bessis (Cherbourg), H. Biessy (La Rochelle), V. Blanc (Antibes-Juan-les-pins), Y. Boucaud-Maitre (Lyon), P. Brunet \& A. Michel (Marseille), B. Cancet (Villeneuve/Lot), J. Carrere (Hyères), A. Cecille (Digneles-bains), G. Chambreuil (La Roche/Yon), P. Chantelat (Vesoul), H. Chardon (Aixen-Provence), C. Charrel (Salon de Provence), H. De Montclos (Bourg-en-Bresse), J. W. Decousser (Dourdan; Rambouillet), J. M. Delarbre/A. Gravet (Mulhouse), D. Deligne (Remiremont), C. Denoix (La Réunion), J. Deregnaucourt (Paris (H. L. Bellon)), F. Desroys du Roure (Chatellerault), S. Dubourdieu (Gisors), Z. El Harrif (Libourne), C. Eloy (Troyes), A. Evers (Annonay), C. Febvre (Montbéliard), D. Fevre (Vienne), S. Gabriel (Monaco), M. J. Galanti (Coulommiers), E. Garnotel (Marseille (HIA Laveran)), M. Gavignet (Lavaur), F. Geffroy (Quimper), G. Grise (ElbeufLouviers), I. Gros (St Denis), I. Hermes (St-Malo), J. Heurte (Beauvais), E. Heusse (Bayeux), D. Jan (Laval), E. Jaouen (Sablé/Sarthe), S. Laluque (Montluçon), R. Lamarca (Narbonne), Laurens (Belfort), A. Le Coustumier (Cahors), E. Lecaillon (Perpignan), C. Lemble (Selestat), M. Leneveu (Poissy; St-Germain), S. Leotard (Grasse), M. N. Letouzey (Villefranche/saone), C. Malbrunot (Corbeil-Essonnes), O. Menouni (Montceau-les-Mines), M. Morel (Le Havre), C. Olive (Fort-de-France), B. Pangon (Versailles), J. G. Paul (Boulogne/mer), J. M. Perez (Pte-à-Pitre), P. Pouedras (Vannes), D. Pressac (Tulle), R. Sanchez (Périgueux), Y. Scat (Pontivy), A. Secher (Dreux), J. Semon (Chalon-sur-Saone), D. Simeon (Langres), C. Simonin (Macon), J. P. Thellier (Château-Thierry), B. Tourand (Alès), A. Vachée (Roubaix), C. Varache (Le Mans), J. Vaucel (St-Brieux), A. C. Vautrin (St-Etienne), A. Verhaeghe (Dunkerke), M. Villemain (Aurillac) and L. Villeneuve (Aubagne).

The work described in this article was presented in part at the 10th International Symposium on Aeromonas and Plesiomonas (Galveston, TX, USA, May 2011).

\section{Author details}

${ }^{1}$ Laboratoire de Bactériologie-Virologie (UMR 5119 - Equipe Pathogènes et Environnements), Université Montpellier 1, 15, Avenue Charles Flahault BP 
14491, 34093 Montpellier Cedex 5, France. ²Laboratoire de Bactériologie, Centre Hospitalier Régional Universitaire de Montpellier (Hôpital Arnaud de Villeneuve), 371, Avenue du Doyen Gaston Giraud, 34295 Montpellier Cedex 5 , France. ${ }^{3}$ Laboratoire d'Hygiène hospitalière, Centre Hospitalier Universitaire de Montpellier, 778, Rue de la Croix Verte, 34000 Montpellier, France. ${ }^{4}$ CNRS UMR 5557 Ecologie microbienne, VetAgro Sup Campus vétérinaire de Lyon, 69280 Marcy-l'Étoile, France. ${ }^{5}$ Groupe d' Etude Français des Aeromonas (GFA), Lyon, France. ${ }^{6} \mathrm{ColBVH}$, Collège de bactériologie, virologie et hygiène des hôpitaux généraux, Lyon, France.

\section{Authors' contributions}

Conceived and designed the study: EJB, HM, BL. Designed and performed the acquisition of clinical data and isolate collection: $\mathrm{ColBVH}, \mathrm{AK}, \mathrm{BL}$ Performed the microbial and molecular genetic analyses: FR (primer design, MLSA and MLPA, PFGE), AK (curator of the clinical isolates collection, rpoB analysis). Analyzed and interpreted the data: FR, BL (all data), HM (PFGE and MLPA), EJB (MLSA), BL (statistics). Drafted the paper: HM, BL. Helped to draft the manuscript: FR. Critically revised the manuscript: EJB. All authors read and approved the final manuscript.

Received: 23 October 2011 Accepted: 30 April 2012

Published: 30 April 2012

\section{References}

1. Janda JM, Abbott SL: The genus Aeromonas: taxonomy, pathogenicity, and infection. Clin Microbiol Rev 2010, 23:35-73.

2. Seshadri R, Joseph SW, Chopra AK, Sha J, Shaw J, Graf J, Haft D, Wu M, Ren Q, Rosovitz MJ, Madupu R, Tallon L, Kim M, Jin S, Vuong H, Stine OC, Ali A, Horneman AJ, Heidelberg JF: Genome sequence of Aeromonas hydrophila ATCC 7966 T: jack of all trades. J Bacteriol 2006, 188:8272-8282.

3. Janda JM, Abbott SL: Evolving concepts regarding the genus Aeromonas: an expanding panorama of species, disease presentations, and unanswered questions. Clin Infect Dis 1998, 27:332-344.

4. Joseph SW, Carnahan AM: Update on the genus Aeromonas. ASM News 2000, 66:218-223.

5. Tonolla M, Demarta A, Peduzzi R: Multilocus genetic relationships between clinical and environmental Aeromonas strains. FEMS Microbiol Lett 1991, 81:193-200

6. Morgan DR, Johnson PC, DuPont HL, Satterwhite TK, Wood LV: Lack of correlation between known virulence properties of Aeromonas hydrophila and enteropathogenicity for humans. Infect Immun 1985, 50:62-65.

7. Kühn I, Albert MJ, Ansaruzzaman M, Bhuiyan NA, Alabi SA, Islam MS, Neogi PK, Huys G, Janssen P, Kersters K, Möllby R: Characterization of Aeromonas spp. isolated from humans with diarrhea, from healthy controls, and from surface water in Bangladesh. J Clin Microbiol 1997, 35:369-373.

8. Albert MJ, Ansaruzzaman M, Talukder KA, Chopra AK, Kuhn I, Rahman M, Faruque AS, Islam MS, Sack RB, Mollby R: Prevalence of enterotoxin genes in Aeromonas spp. isolated from children with diarrhea, healthy controls, and the environment. J Clin Microbiol 2000, 3790:3785.

9. Romano S, Aujoulat F, Jumas-Bilak E, Masnou A, Jeannot J-L, Falsen E, Marchandin $\mathrm{H}$, Teyssier $\mathrm{C}$ : Multilocus sequence typing supports the hypothesis that Ochrobactrum anthropi displays a human-associated subpopulation. BMC Microbiol 2009, 9:267.

10. van Mansfeld R, Jongerden I, Bootsma M, Buiting A, Bonten M, Willems R: The population genetics of Pseudomonas aeruginosa isolates from different patient populations exhibits high-level host specificity. PLoS One 2010, 5:e13482.

11. Aujoulat F, Jumas-Bilak E, Masnou A, Sallé F, Faure D, Segonds C, Marchandin $H$, Teyssier C: Multilocus sequence-based analysis delineates a clonal population of Agrobacterium (Rhizobium) radiobacter (Agrobacterium tumefaciens) of human origin. J Bacteriol 2011, 193:2608-2618.

12. Bidet $\mathrm{P}$, Mahjoub-Messai F, Blanco J, Blanco J, Dehem M, Aujard Y, Bingen $E$, Bonacorsi S: Combined multilocus sequence typing and $\mathrm{O}$ serogrouping distinguishes Escherichia coli subtypes associated with infant urosepsis and/or meningitis. J Inf Dis 2007, 196:297-303.

13. Hoffmaster AR, Novak RT, Marston CK, Gee JE, Helsel L, Pruckler JM, Wilkins PP: Genetic diversity of clinical isolates of Bacillus cereus using multilocus sequence typing. BMC Microbiol 2008, 8:191.
14. Kaiser S, Biehler K, Jonas D: A Stenotrophomonas maltophilia multilocus sequence typing scheme for inferring population structure. J Bacteriol 2009, 191:2934-2943.

15. Martino ME, Fasolato L, Montemurro F, Rosteghin M, Manfrin A, Patarnello T, Novelli E, Cardazzo B: Determination of microbial diversity of aeromonas strains on the basis of multilocus sequence typing, phenotype, and presence of putative virulence genes. Appl Environ Microbiol 2011, 77:4986-5000

16. Martinez-Murcia AJ, Monera A, Saavedra MJ, Oncina R, Lopez-Alvarez M, Lara E, Figueras MJ: Multilocus phylogenetic analysis of the genus Aeromonas. Syst Appl Microbiol 2011, 34:189-199.

17. Lamy B, Kodjo A, Laurent F: Prospective nationwide study of Aeromonas infections in France. J Clin Microbiol 2009, 47:1234-1237.

18. Miranda G, Kelly C, Solorzano F, Leanos B, Coria R, Patterson JE: Use of pulsed-field gel electrophoresis typing to study an outbreak of infection due to Serratia marcescens in a neonatal intensive care unit. J Clin Microbiol 1996, 34:3138-3141.

19. BioEdit: Sequence alignment editor for Windows 95/98/NT/XP [http:// www.mbio.ncsu.edu/bioedit/bioedit.html].

20. Felsenstein J: Distance methods for inferring phylogenies: a justification. Evolution 1984, 38:16-24.

21. Guindon S, Gascuel O: A simple, fast, and accurate algorithm to estimate large phylogenies by maximum likelihood. Syst Biol 2003, 52:696-704.

22. Posada D: jModelTest: phylogenetic model averaging. Mol Biol Evol 2008, 25:1253-1256.

23. Feil EJ, Li BC, Aanensen DM, Hanage WP, Spratt BG: eBURST: inferring patterns of evolutionary descent among clusters of related bacterial genotypes from multilocus sequence typing data. J Bacteriol 2004, 186:1518-1530.

24. Haubold B, Hudson RR: LIAN 3.0: detecting linkage disequilibrium in multilocus data. Linkage analysis. Bioinformatics 2000, 16:847-848.

25. Jolley KA, Feil EJ, Chan MS, Maiden MC: Sequence type analysis and recombinational tests (START). Bioinformatics 2001, 17:1230-1231.

26. Huson DH, Bryant D: Application of phylogenetic networks in evolutionary studies. Mol Biol Evol 2006, 23:254-267.

27. Martin DP, Lemey P, Lott M, Moulton V, Posada D, Lefeuvre P: RDP3: a flexible and fast computer program for analyzing recombination. Bioinformatics 2010, 26:2462-2463.

28. Silver AC, Williams D, Faucher J, Horneman AJ, Gogarten JP, Graf J: Complex evolutionary history of the Aeromonas veronii group revealed by host interaction and DNA sequence data. PLoS One 2011, 6:e16751.

29. Khan NH, Ahsan M, Yoshizawa S, Hosoya S, Yokota A, Kogure K: Multilocus sequence typing and phylogenetic analyses of Pseudomonas aeruginosa isolates from the ocean. Appl Env Microbiol 2008, 74:6194-6205.

30. Jolley KA, Maiden MC: BIGSdb: scalable analysis of bacterial genome variation at the population level. BMC Bioinformatics 2010, 11:595.

31. Beatson SA, das Graças de Luna M, Bachmann NL, Alikhan N-F, Hanks KR, Sullivan MJ, Wee BA, Freitas-Almeida AC, Dos Santos PA, de Melo JTB, Squire DJP, Cunningham AF, Fitzgerald JR, Henderson IR: Genome sequence of the emerging pathogen Aeromonas caviae. J Bacteriol 2011, 193:1286-1287.

32. Li Y, Liu Y, Zhou Z, Huang H, Ren Y, Zhang Y, Li G, Zhou Z, Wang L: Complete genome sequence of Aeromonas veronii strain B565.J Bacteriol 2011, 193:3389-3390.

33. Reith ME, Singh RK, Curtis B, Boyd JM, Bouevitch A, Kimball J, Munholland J, Murphy C, Sarty D, Williams J, Nash JH, Johnson SC, Brown LL: The genome of Aeromonas salmonicida subsp. salmonicida A449: insights into the evolution of a fish pathogen. BMC Genomics 2008, 9:427

34. van Berkum P, Elia P, Eardly BD: Multilocus sequence typing as an approach for population analysis of Medicago-nodulating rhizobia. $J$ Bacteriol 2006, 188:5570-5577.

35. Pirnay J-P, Matthijs S, Colak H, Chablain P, Bilocq F, Van Eldere J, De Vos D, Zizi M, Triest L, Cornelis P: Global Pseudomonas aeruginosa biodiversity as reflected in a Belgian river. Env Microbiol 2005, 7:969-980.

36. Aguilera-Arreola MG, Hernández-Rodríguez C, Zúñiga G, Figueras MJ, Garduño RA, Castro-Escarpulli G: Virulence potential and genetic diversity of Aeromonas caviae, Aeromonas veronii, and Aeromonas hydrophila clinical isolates from Mexico and Spain: a comparative study. Can 」 Microbiol 2007, 53:877-887.

37. Sneath PHA: Evidence from Aeromonas for genetic crossing-over in ribosomal sequences. Int J Syst Bacteriol 1993, 43:626-629. 
38. Morandi A, Zhaxybayeva O, Gogarten JP, Graf J: Evolutionary and diagnostic implications of intragenomic heterogeneity in the 16 S rRNA gene in Aeromonas strains. J Bacteriol 2005, 187:6561-6564.

39. Umelo E, Trust TJ: Physical map of the chromosome of Aeromonas salmonicida and genomic comparisons between Aeromonas strains. Microbiol 1998, 144(8):2141-2149.

40. Georgiades K, Raoult D: Defining pathogenic bacterial species in the genomic era. Front Microbiol 2010, 1:151.

41. Martinez-Murcia AJ, Benlloch S, Collins MD: Phylogenetic interrelationships of members of the genera Aeromonas and Plesiomonas as determined by $16 \mathrm{~S}$ ribosomal DNA sequencing: Lack of congruence with results of DNA-DNA hybridizations. Int J Syst Bacterio/ 1992, 42:412-421.

42. Huys $G$, Kämpfer $P$, Swings J: New DNA-DNA hybridization and phenotypic data on the species Aeromonas ichthiosmia and Aeromonas allosaccharophila: Aeromonas ichthiosmia Schubert et al. 1990 is a later synonym of Aeromonas veronii Hickman-Brenner et al. 1987. Syst Appl Microbiol 2001, 24:177-182.

43. Nhung PH, Hata H, Ohkusu K, Noda M, Shah MM, Goto K, Ezaki T: Use of the novel phylogenetic marker dnaJ and DNA-DNA hybridization to clarify interrelationships within the genus Aeromonas. Int J Syst Evol Microbiol 2007, 57:1232-1237

44. Saavedra MJ, Perea V, Fontes MC, Martins C, Martínez-Murcia A: Phylogenetic identification of Aeromonas strains isolated from carcasses of pig as new members of the species Aeromonas allosaccharophila. Antonie Van Leeuwenhoek 2007, 91:159-167.

45. Miñana-Galbis D, Urbizu-Serrano A, Farfán M, Fusté MC, Lorén JG: Phylogenetic analysis and identification of Aeromonas species based on sequencing of the cpn60 universal target. Int J Syst Evol Microbiol 2009, 59:1976-1983.

46. Vial L, Chapalain A, Groleau M, Déziel E: The various lifestyles of the Burkholderia cepacia complex species: a tribute to adaptation. Env Microbiol 2011, 13:1-12.

47. Monfort P, Baleux B: Dynamics of Aeromonas hydrophila, Aeromonas sobria and Aeromonas caviae in a sewage treatment pond. Appl Env Microbiol 1990, 56:1999-2006.

48. Goñi-Urriza M, Capdepuy M, Arpin C, Raymond N, Caumette P, Quentin C Impact of an urban effluent on antibiotic resistance of riverine Enterobacteriaceae and Aeromonas spp. Appl Env Microbiol 2000, 66:125-132.

49. Alperi A, Martinez-Murcia AJ, Ko WC, Monera A, Saavedra MJ, Figueras MJ Aeromonas taiwanensis sp. nov. and Aeromonas sanarellii sp. nov., clinical species from Taiwan. Int J Syst Evol Microbiol 2009, 60:2048-2055.

50. Alperi A, Martinez-Murcia AJ, Monera A, Saavedra MJ, Figueras MJ: Aeromonas fluvialis sp. nov., isolated from a Spanish river. Int J Syst Evol Microbiol 2009, 60:72-77.

51. Miñana-Galbis D, Farfán M, Gaspar Lorén J, Carmen Fusté M: Proposal to assign Aeromonas diversa sp. nov. as a novel species designation for Aeromonas group 501. Syst Appl Microbiol 2010, 33:15-19.

52. Martinez-Murcia AJ, Saavedra MJ, Mota VR, Maier T, Stackebrandt E, Cousin S: Aeromonas aquariorum sp. nov., isolated from aquaria of ornamental fish. Int J Syst Evol Microbiol 2008, 58:1169-1175.

53. Lamy B, Laurent $F$, Kodjo A: Validation of a partial rpoB gene sequence as a tool for phylogenetic identification of aeromonads isolated from environmental sources. Can J Microbiol 2010, 56:217-228.

54. Esteve C, Gutierrez MC, Ventosa A: DNA relatedness among Aeromonas allosaccharophila strains and DNA hybridization groups of the genus Aeromonas. Int J Syst Bacteriol 1995, 45:390-391.

55. Saha P, Chakrabarti T: Aeromonas sharmana sp. nov., isolated from a warm spring. Int J Syst Evol Microbiol 2006, 56:1905-1909.

56. Martínez-Murcia AJ, Figueras MJ, Saavedra MJ, Stackebrandt E: The recently proposed species Aeromonas sharmana sp. nov., isolate GPTSA-6 T, is not a member of the genus Aeromonas. Int Microbiol 2007, 10:61-64.

doi:10.1186/1471-2180-12-62

Cite this article as: Roger et al:: Multilocus genetics to reconstruct aeromonad evolution. BMC Microbiology 2012 12:62.

\section{Submit your next manuscript to BioMed Central and take full advantage of:}

- Convenient online submission

- Thorough peer review

- No space constraints or color figure charges

- Immediate publication on acceptance

- Inclusion in PubMed, CAS, Scopus and Google Scholar

- Research which is freely available for redistribution

Submit your manuscript at www.biomedcentral.com/submit 